\title{
COMPOSITE QUARKS AND LEPTONS
}

W. Buchmiller

CERN - Geneva

\begin{abstract}
1) Introduction
2) A preon model of Pati-Salam type

3) Bounds on the substructure scale

4) Composite $W$-bosons

5) Massless composite scalars, fermions and vector bosons

6) Quasi-Goldstone fermions

7) Composite Higgs fields and exceptional coset space

8) Conclusions
\end{abstract}

Lectures given at the XXIV Internationale Universitätswochen für Kernphysik Schladming, Austria

20 February - 1 March 1985

CERN-TH. $4189 / 85$

May 1985 


\section{INTRODUCTION}

The present theory of particle physics is the standard model of strong and electroweak interactions [1]. It is a renormalizable quantum field theory which describes sucessfully all our experimental information about the structure of matter down to distances of $10^{-16} \mathrm{~cm}$. Despite its extraordinary success, however, there appears to be a general consensus that the standard model can only be an effective "low energy" Lagrangian of a more fundamental theory. Indeed, the gauge structure of strong, electromagnetic and weak interactions suggests a unification of all forces $[2,3]$, the replication of families and the hierachical pattern of fermion masses point towards a common underlying structure of quarks and leptons, and the "naturalness problem" [4-6] of fundamental scalar fields seems to require a modification of the Higgs sector.

In these lectures we will discuss the current status of attempts to improve some of the unsatisfactory features of the standard model by making quarks, leptons, Higgs bosons and possibly also the vector bosons of the weak interactions composite. Despite considerable efforts [7] in recent years there exists at present no satisfactory "standard composite model" which would provide a convincing explanation of the family replication and the quark-lepton mass sepctrum and which could be distinguished from the standard model through definite, experimentally testable predictions. We will therefore not discuss any specific model in detail but rather concentrate on the general features which characterize the different classes of composite models.

In sec.2 we illustrate the role of global and local symmetries by means of a preon model of pati-salam type. Sec. 3 is devoted to the bounds which high energy scattering experiments and rare processes impose on the substructure 
scale $\Lambda$. In sec. 4 we discuss composite $W$-bosons, which occur in preon models where the substructure scale is of the order of the Fermi scale, $\Lambda \sim_{F}^{-1 / 2}$, and which represent an alternative to the standard gauge theory of weak interactions. As an example of the rich phenomenology of such models with "nearby compositeness" we describe the signatures of light pseudoscalar leptoquarks. Sec.5 deals with theoretical constraints on the dynamics of (almost) massless composite particles: the effective Lagrangian for Goldstone bosons, the 't Hooft consistency conditions for chiral fermions and the the Case-Gasiorowicz-Weinberg-Witten theorem for massless vector bosons. In sec. 6 we discuss the special features of supersymmetric (SUSY) preon models, in particular the natural appearance of massless composite fermions through the Goldstone mechanism. An example of the resulting SUSY $\sigma$-models and the particularly interesting coset spaces of exceptional groups are described in sec.7, and finally we present some conclusions in sec. 8 .

Before we describe attempts to improve unsatisfactory features of the standard model, however, let us recall the basic ingredients of the Glashow-Weinberg-Salam theory [8]. The strong and electroweak interactions are mediated through the exchange of gauge bosons $G_{\mu}^{A}(A=1, \ldots, 8), W_{\mu}^{I}(I=1, \ldots, 3)$ and $B_{\mu}$ which are contained in the gauge group

$G_{S M}=S U(3)_{C} \times S U(2)_{W} \times U(1)_{Y} \cdot$

There are three families of quarks and leptons

$$
\begin{aligned}
& q_{L}^{\alpha_{i}}=\left(\begin{array}{c}
u_{L}^{\alpha i} \\
a_{L}^{\alpha i}
\end{array}\right), \quad u_{R}^{\alpha i}, a_{R}^{\alpha_{i}}, \\
& I_{L}^{i}=\left(\begin{array}{c}
v_{L}^{i} \\
e_{L}^{-i}
\end{array}\right), \quad\left(v_{R}^{i}\right), e_{R}^{-i},
\end{aligned}
$$


with colour, weak isospin and hypercharge quantum numbers

$$
\begin{aligned}
& q_{L} \sim(3,2)_{\frac{1}{6}}, \quad u_{R} \sim(3,1)_{\frac{2}{3}}, \quad d_{R} \sim(3,1)_{-\frac{1}{3}}, \\
& l_{L} \sim(1,2)_{\simeq \frac{1}{2}}, \quad\left(v_{R} \sim(1,1)_{0}\right), \quad e_{R} \sim(1,1)_{-1}
\end{aligned}
$$

where $\alpha=1, \ldots, 3$ is a colour index and $i=1, \ldots, 3$ a generation index for the $e-, \mu$ - and $\tau$-families. Note that left-handed fermions are $\mathrm{SU}(2)_{\mathrm{W}}$ doublets whereas right-handed fermions are $\mathrm{SU}(2)_{\mathrm{W}}$ singlets. So far there is no experimental evidence for righthanded neutrinos $\nu_{R}$ [9] which play a special role because they do not couple to gauge bosons. The spontaneous breaking of $S U(2)_{W} \times U(1)_{Y}$ requires scalar fields, such as

$$
\phi_{1}=\left(\begin{array}{l}
\phi_{1}^{0} \\
\phi_{1}^{-}
\end{array}\right) \sim(1,2)-\frac{1}{2}
$$

and

$\phi_{2}=\left(\begin{array}{c}\phi^{+} \\ 2 \\ \phi^{0} \\ 2\end{array}\right) \sim(1,2) \frac{1}{2}$,

where the superscripts denote the electric charges which are the sum of hypercharge and weak isospin, i.e., $Q=Y+T_{3}$. In the minimal model the second Higgs doublet is not an independent field but related to $\phi_{1}$ through $\phi_{2}^{p}=\varepsilon^{p q} \phi_{1}^{*} q^{*}$

Given the $S U(3)_{C} \times S U(2)_{W} \times U(1)_{Y}$ quantum numbers of quark, lepton and Higgs fields their couplings to the gauge bosons $G_{\mu}^{A}, W_{\mu}^{I}$ and $B_{\mu}$ are entirely determined up to three 
universal gauge coupling constants $g_{3}, g_{2}$ and $g_{1}$. On the contrary the couplings between fermions and scalars are largely arbitrary. The most general renormalizable, SU(3) $x$ SU(2) $\times U$ (1) invariant Lagrangian is given by

$$
\begin{aligned}
L_{Y}= & g(u) \underset{j}{i} \bar{q}_{L i} \phi_{1} u_{R}^{j}+g(d) \underset{j}{i} \bar{q}_{L i} \phi_{2} d_{R}^{j} \\
& \left(+g(v) i \bar{j}_{L i} \phi_{1} v_{R}^{j}\right)+g
\end{aligned}
$$

and involves 3 unconstrained complex $3 \times 3$ matrices of Yukawa couplings.

In the standard model the effective potential of the Higgs fields $\phi_{1}$ and $\phi_{2}$ has a minimum which braks $s U(2)_{W} x$ $\mathrm{X} U(1)_{\mathrm{Y}}$ to $\mathrm{U}(1)_{\mathrm{EM}}$ :

$\left.<\phi_{1}\right\rangle_{0}=\left(\begin{array}{c}v_{1} \\ 0\end{array}\right), \quad<\phi_{2}>_{0}=\left(\begin{array}{c}0 \\ v_{2}\end{array}\right)$.

This spontaneous symmetry breaking leads to masses for the $\mathrm{W}^{ \pm}$and $\mathrm{Z}$ vector bosons:

$\mathrm{M}_{\mathrm{W}}^{2}=\frac{1}{2} \mathrm{~g}_{2}^{2}\left(\mathrm{v}_{1}^{2}+\mathrm{v}_{2}^{2}\right), \quad \mathrm{M}_{\mathrm{Z}}^{2}=\frac{1}{2}\left(\mathrm{~g}_{1}^{2}+\mathrm{g}_{2}^{2}\right)\left(\mathrm{v}_{1}^{2}+\mathrm{v}_{2}^{2}\right)$,

$v_{1}^{2}+v_{2}^{2}=\left(2 \sqrt{2} G_{F}\right)^{-1}=(174 \mathrm{GeV})^{2}$.

Furthermore one obtaines from equs. (5) and (6) mass matrices for $u$ - and d-type quarks and for the charged leptons.

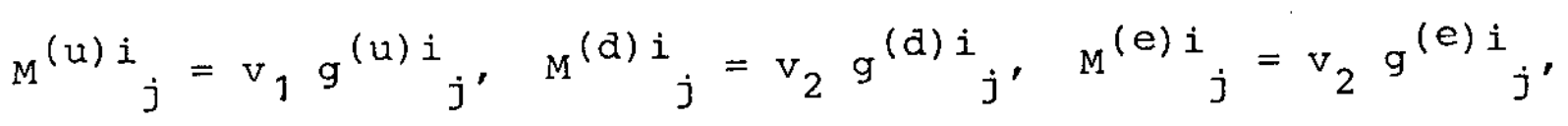

which yield the mass eigenvalues $m_{e} \ldots, m_{t}$ as well as the parameters $\theta_{1}, \Theta_{2}, \theta_{3}$ and $\delta$ of the Kobayashi-Maskawa matrix. 
The structure of the matter sector of the standard model, as described above, provokes the following questions:

(i) Why is there a threefold replication of quark-lepton generations?

(ii) What is the dynamical origin of symmetry breaking and fermion masses?

It is indeed difficult to believe that the appearence of three quark-lepton families with identical $\mathrm{SU}(3){ }_{\mathrm{C}} \times \mathrm{SU}(2)_{\mathrm{W}} \mathrm{x}$ $x U(1)_{Y}$ quantum numbers is a mere accident and that yukawa couplings which, from the electron to the b-quark, vary over four orders of magnitude should be considered as fundamental parameters like the gauge couplings. Furthermore it is obvious that an understanding of the Yukawa couplings is impossible within the standard model and that some new structure is required. In analogy to hadron physics, where the pion-nucleon coupling constant can be computed (at least in principle) within $Q C D$, it is natural to investigate the possibility that also quarks, leptons and Higgs bosons are composite. The Lagrangian (5) would then be valid only at momentum transfers small compared to the substructure scale $\Lambda$ and at distances $x<\frac{1}{\Lambda}$ the full structure of the three-point functions would become visible (cf.Fig.1). It is conceivable that such a preon theory
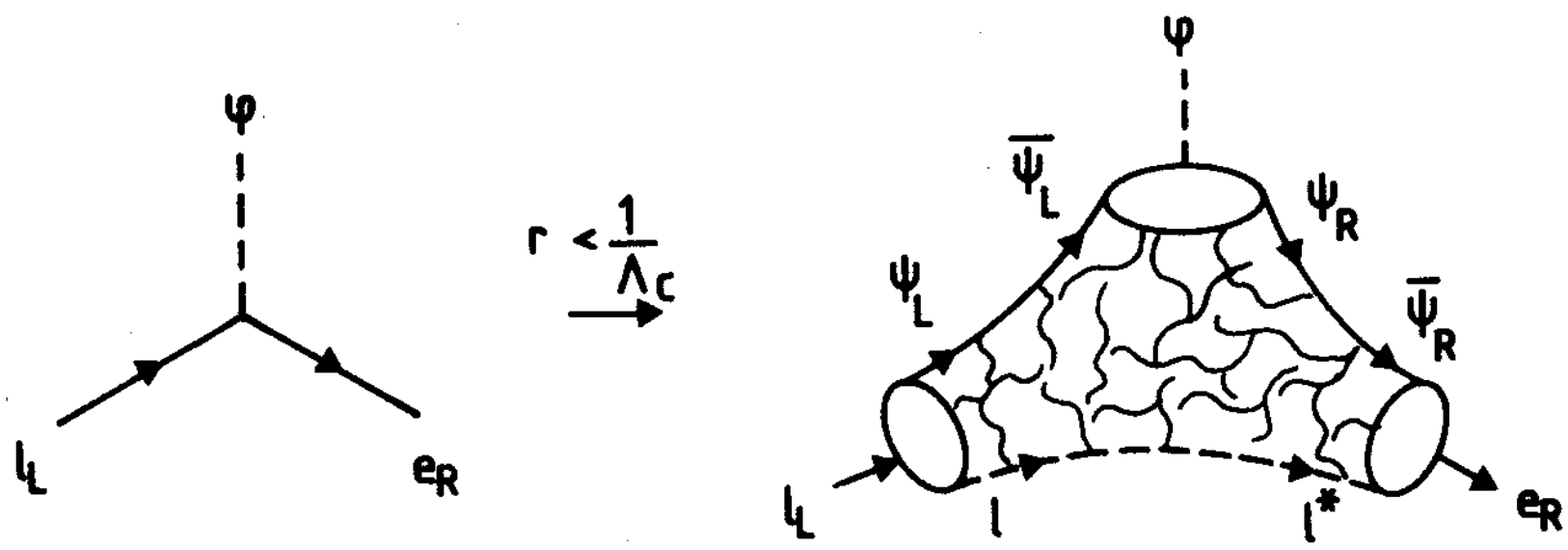

Fig. 1.: Structure of Yukawa coupling in preon theory at distances $r<\frac{1}{\Lambda}$. 
could explain generations as some sort of excitations and that the fermion mass matrices would be calculable (at least in principle) in terms of a few parameters.

As the desire to understand the fermion mass spectrum is one of the main motivations for quark-lepton substructure, let us briefly recall some basic facts of the phenomenology of quark mass matrices [10]. The mass matrices $M^{(u)}$ and $\mathrm{M}^{(\mathrm{d})}$ can be diagonalized by bi-unitary transformations [ 8 ]

$$
\begin{aligned}
& U_{L}^{+} M^{(u)} U_{R}=M_{D}^{(u)}=\left(\begin{array}{lll}
m_{u} & & \\
& m_{C} & \\
& & m_{t}
\end{array}\right), \quad U_{L(R)}^{+} U_{L(R)}=1, \\
& V_{L}^{+}{ }^{(d)} V_{R}=M_{D}^{(d)}=\left(\begin{array}{lll}
m_{d} & \\
& m_{s} & \\
& & m_{b}
\end{array}\right), \\
&
\end{aligned}
$$

which yield the Kobayashi-Maskawa matrix as

$\mathrm{K}=\mathrm{V}_{\mathrm{L}}^{+} \mathrm{U}_{\mathrm{L}}$

Wolfenstein has suggested an interesting parametrization of $\mathrm{K}$ [11] which is consistent with all experimental information:
d
s
$\mathrm{b}$
$\mathrm{K}=\quad \mathrm{c}\left(\begin{array}{ccc}1-\frac{\lambda^{2}}{2} & \lambda & \lambda^{3}\left(\rho-i_{n}\right) \\ -\lambda & 1-\frac{\lambda^{2}}{2} & \lambda^{2} \mathrm{~A} \\ \lambda^{2} \mathrm{~A}\left(1-\rho-i_{\eta}\right) & -\lambda^{2} \mathrm{~A} & 1\end{array}\right)+o\left(\lambda^{4}\right)$.

(11)

Here the cosine of the Cabibbo angle $\lambda=\cos \theta_{C} \hat{\lambda}_{0} 0.22$ plays the role of an expansion parameter, and $A, \rho$, and $\eta$ are real 
numbers of order one.

Recently Frampton and Jarlskog have shown that in a basis where the mass matrices are hermitean they satisfy the relation [12]

$\frac{1}{m_{t}} M^{(u)}=\frac{1}{m_{b}} M^{(d)}+O\left(\lambda^{2}\right)$,

a regularity pointing towards a common origin of up- and down- quark masses. It is even possible to find a basis where one has

$\frac{1}{m_{t}} M^{(u)}=\left(\begin{array}{lll}0 & 0 & \\ & & 1\end{array}\right)+O\left(\lambda^{2}\right)=\frac{1}{m_{b}} M^{(d)}$.

This is achieved by choosing the fields $q_{L}^{i}, u_{R}^{i}$ and $d_{R}^{i}$ such that $V_{L}=V_{R}=U_{R}=1$, which implies

$\mathrm{K}^{\prime}=\mathrm{U}_{\mathrm{L}}$

In this basis $M^{(d)}$ is diagonal and one has $\left(\lambda^{2} \approx \frac{1}{20}\right)$

$\frac{1}{m_{b}} M^{(d)}=\left(\begin{array}{lll}\frac{m_{d}}{m_{b}} & & \\ & \frac{m_{s}}{m_{b}} & \\ & & 1\end{array}\right)=\left(\begin{array}{lll}0 & & \\ & 0 & 1\end{array}\right)+o\left(\lambda^{2}\right)$,

where we have used $m_{d} / m_{b} \approx 1 / 600, m_{s} / m_{b} \approx 1 / 30$ [13]. For the up-quark mass matrix one obtains from equs. (9)-(11) and with $m_{u} / m_{t}<1 / 4000, m_{c} / m_{t}<1 / 18$ (cf. [13], $m_{t}>20 \mathrm{GeV}$ ):

$\frac{1}{m_{t}} M^{(u)}=K\left(\begin{array}{ccc}\frac{m_{u}}{m_{t}} & & \\ & \frac{m_{c}}{m_{t}} & \\ & & 1\end{array}\right)=\left(\begin{array}{lll}0 & \\ & 0 \\ & & 1\end{array}\right)+o\left(\lambda^{2}\right)$. 
Equs. (12) and (13) suggest that, as many authors have speculated, the quark mass matrices may be calculable in some kind of perturbation theory, starting from

$\frac{1}{m_{b}} M_{(0)}^{(d)}=\frac{1}{m_{t}} \quad M_{(0)}^{(u)}=\left(\begin{array}{lll}0 & & \\ & 0 & \\ & & 1\end{array}\right)$.

The different mass scales for $u$ - and d-type quark masses could be due to different vacuum expectation values of the two Higgs doublets $\mathrm{H}_{1}$ and $\mathrm{H}_{2}$ :

$\frac{m_{b}}{m_{t}} \approx \frac{v_{2}}{v_{1}} \approx \frac{1}{10}$

The goal of composite models is to understand the structure of the quark and lepton mass matrices and to compute eventually the parameters $v_{1}, v_{2}, \lambda, \ldots$ from a more fundamental preon theory.

\section{A PREON MODEL OF PATI-SALAM TYPE}

Pati and Salam were the first to speculate that quarks and leptons may have a further substructure [14]. They were led to this conjecture by the symmetries of the quark-lepton spectrum and in this section we will follow their approach. Early preon models can be found in [15]-[17] and a more complete list of references in the reviews [18]-[20]. There is an interesting class of composite models with only fermionic preons, such as the models of Harari [21], Shupe [22], Harari and Seiberg [23], Bars [24] and Schrempp and Schrempp [25], as well as the recent work on "composite Higgs models" [26], which we will not discuss. We will focus on fermion-boson models which have a simple 
quantum number structure. One may object that fundamental scalar fields are "unnatural" [4-6] but, as we will see in sections 6 and 7 , this problem does not exist in supersymmetric models which we consider to be the most promising candidates for a theory of quark-lepton substructure. The content of this section is closely related to the model of Barbieri, Masiero and Mahapatra [27], similar models can be found in [28]-[31].

It is very instructive to study the dependence of the global symmetries of the standard model on the Higgs part of the Lagrangian (cf.(5)). For arbitrary Yukawa couplings $g(u, d, v, e) i_{j} \neq 0$ one has

$G=S U(3)_{C} \times S U(2)_{W} \times U(1)^{3}$,

with

$U(1)^{3}=U(1)_{q} \times U(1)_{\ell} \times U(1)_{\phi}$.

The U(1) charges of quarks, leptons and Higgs fields are given in table I. One linear combination, the hypercharge $\mathrm{Y}$, is gauged together with

Table I.: U(1) charges of quarks, leptons and Higgs scalars.

\begin{tabular}{|c|c|c|c|c|c|c|c|c|}
\hline & ${ }^{q_{L}}$ & ${ }^{\ell} L$ & $\phi_{1}$ & $\mathrm{u}_{\ell}$ & $\mathrm{d}_{\mathrm{R}}$ & ${ }^{\nu_{R}}$ & ${ }^{e}{ }_{R}$ & $\phi_{2}$ \\
\hline $\mathrm{Q}_{\mathrm{q}}$ & 1 & 0 & 0 & 1 & 1 & 0 & 0 & 0 \\
\hline $\mathrm{Q}_{\ell}$ & 0 & 1 & 0 & 0 & 0 & 0 & 1 & 0 \\
\hline $\mathrm{Q}_{\phi}$ & 0 & 0 & 1 & -1 & 1 & -1 & 1 & -1 \\
\hline
\end{tabular}


colour and weak isospin. The two remaining U(1) factors correspond to baryon and lepton number:

$$
\begin{aligned}
& Y=\frac{1}{6} Q_{q}-\frac{1}{2} Q_{\ell}-\frac{1}{2} Q_{\phi}, \\
& B=\frac{1}{3} Q_{q}, \\
& L=Q_{\ell} .
\end{aligned}
$$

It is a very remarkable feature of the standard model Lagrangian that it contains no global symmetries beyond baryon and lepton number. This changes drastically in the extreme case of vanishing Yukawa couplings, $g(u, d, v, e) i_{j}=0$. In this case there is no difference between the three families and one gains a global $U(3)$ invariance for each irreducible $S U(3)_{C} \times S U(2)_{W} \times U(1)_{Y}$ representation (cf.(3)), i.e.,

$\mathrm{G}^{\prime}=\mathrm{SU}(3)_{\mathrm{C}} \times \mathrm{SU}(2)_{\mathrm{W}} \times \mathrm{U}^{(1)_{\mathrm{Y}}} \times \mathrm{U}^{(3)^{6}}$

Note that in the absence of Yukawa couplings the right handed neutrinos are free particles. The huge symmetry G' has to be broken to $G$ in order to generate masses for quarks and leptons. To understand how this happens is one of the big problems of composite models where one wants to calculate fermion masses starting, in first approximation, from three massless families of quarks and leptons.

Many preon models are based on the global symmetry which one obtains for equal Yukawa couplings, $g^{(u)}=g^{(d)}=g^{(v)}=g^{(e)} \equiv g$, in the case of one family. Then the Lagrangian (5) becomes

$$
L_{H}=g(u) \bar{q}_{L p} \phi p_{1}^{p} u_{R}+g(d) \bar{q}_{L p} \phi \frac{p}{2} d_{R}+
$$


$+g(\nu) \bar{l}_{L p} \phi p^{p} \nu_{R}+g(e) \bar{q}_{L p} \phi \frac{p}{2} e_{R}+c . c$.

$=g \bar{f}_{L p} \phi_{s}^{p_{f}^{s}}{ }_{R}+$ c.c. ,

with

$f_{L}^{p}=\left(q_{L}^{\alpha p}, e_{L}^{p}\right), f_{R}^{S}=\left(\begin{array}{cc}u_{R}^{\alpha} & v_{R} \\ \alpha_{R}^{\alpha} & e_{R}^{-}\end{array}\right)$,

$\phi_{\mathrm{S}}^{\mathrm{p}}=\left(\phi_{1}^{\mathrm{p}}, \phi_{2}^{\mathrm{p}}\right)$

Obviously the Lagrangian (22) has the symmetry

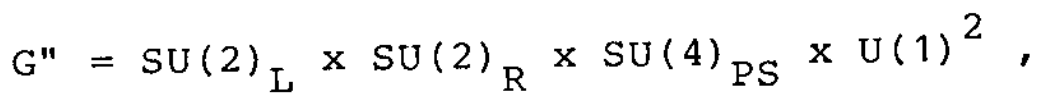

under which fermions and scalars transform as

$f_{L} \sim(2,1,4), \quad f_{R} \sim(1,2,4), \quad \phi \sim(2,2,1)$.

The Pati-Salam symmetry SU(4) PS contains lepton number as "fourth colour" [2]. It appears here as a global symmetry which, in the limit of vanishing gauge couplings $g_{1}$ and $g_{3}$, is an exact invariance of the whole Lagrangian. An attractive feature of the SU(4) PS symmetry is that it contains B-L. The three diagonal $\mathrm{SU}(4) \lambda$-matrices are $\lambda_{3}$ and

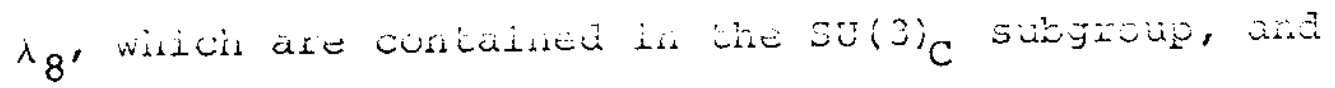

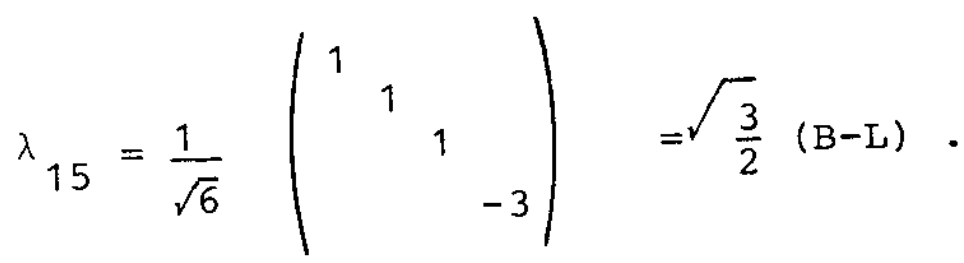


The electric charge takes the symmetric form

$Q=\frac{1}{2}(B-L)+T_{3 L}+T_{3 R}$,

where $\mathrm{T}_{3 \mathrm{~L}(\mathrm{R})}$ are the diagonal generators of $\mathrm{SU}(2) \mathrm{L}(\mathrm{R})$.

The fermion representation (24) is chiral (i.e.,

different for left and right handed fermions) with respect to $\mathrm{SU}(2)_{\mathrm{L}} \times \mathrm{SU}(2)_{\mathrm{R}^{\prime}}$ and vectorlike (i.e., identical for left and right handed fermions) with respect to SU(4) PS Therefore it is natural, to associate in a fermion-boson composite model the $\mathrm{SU}(2)_{\mathrm{L}} \times \mathrm{SU}(2)_{\mathrm{R}}$ subgroup with fermionic preons and the SU(4) subgroup with scalar preons [14]. We thus introduce as preons the fermions

$\psi_{\mathrm{I}}^{\mathrm{P}} \sim(2,1,1), \quad \psi_{\mathrm{R}}^{\mathrm{S}} \sim(1,2,1)$

and the scalars

$S=\left(h^{\alpha}, l\right) \sim(1,1,4)$

with $\mathrm{SU}(3)_{C} \times \mathrm{SU}(2)_{\mathrm{W}} \times \mathrm{U}(1)_{\mathrm{Y}}$ gauge quantum numbers

$\psi_{\mathrm{L}} \sim(1,2) \mathrm{O}^{\prime} \quad \psi_{\mathrm{R}}^{1} \sim(1,1)_{\frac{1}{2}}, \quad \psi_{\mathrm{R}}^{2} \sim(1,1)_{-\frac{1}{2}}$,

$h \sim(3,1)_{\frac{1}{6}}, \quad \ell \sim(1,1)_{-\frac{1}{2}}$

The most common choice for the binding force is a confirning "hypercolour" gauge interaction with gauge group $\mathrm{SU}(\mathrm{n}) \mathrm{HC}$ under which the preons transform like 
$\psi_{L, R} \sim \underline{\mathrm{n}}, \quad s \sim \underline{\underline{n}}^{*}$.

Equs. (27) - (29) define a preon theory whose bound state spectrum is in principle calculable. Unfortunately the dynamics of confining gauge theories is very complicated and currently no rigorous results are known for theories with fermions and scalars in the fundamental representation of the gauge group. In analogy with QCD one expects that the simplest hypercolour singlet composite operators with different "flavour" quantum numbers create different physical states from the vacuum. This yields quarks and leptons as fermion-boson bound states,

$$
\begin{array}{lll}
q_{L}=\left(\psi_{L} h\right), & u_{R}=\left(\psi_{R}^{1} h\right), & d_{R}=\left(\psi_{R}^{2} h\right), \\
\ell_{L}=\left(\psi_{L}^{\ell}\right), & v_{R}=\left(\psi_{R}^{1} \ell\right), & e_{R}^{-}=\left(\psi_{R}^{2} \ell\right),
\end{array}
$$

and the Higgs scalars as fermion-antifermion bound states,

$\phi_{\mathrm{S}}^{\mathrm{P}}=\left(\bar{\psi}_{\mathrm{RS}}{ }_{\mathrm{L}}^{\mathrm{P}}\right)$

Vacuum expectation values of the composite scalars $\phi_{S}^{P}$,

$\left\langle\phi_{\mathrm{S}}^{\mathrm{P}}\right\rangle=\left\langle\bar{\psi}_{\mathrm{RS}} \psi_{\mathrm{L}}^{\mathrm{P}}\right\rangle=\mathrm{v} \delta_{\mathrm{S}}^{\mathrm{P}}$,

can lead to the technicolour-type [5] symmetry breaking $\mathrm{SU}(2)_{\mathrm{L}} \times \mathrm{SU}(2)_{\mathrm{R}} \rightarrow \mathrm{SU}(2)_{\mathrm{D}}$ and thereby give masses to the W-bosons. 
In addition to the wanted bound states of equs. (30) and (31) one expects composite vector and scalar bosons corresponding to the following operators $\left(S U(2){ }_{L} \times S U(2){ }_{R} x\right.$ $x$ SU(4) $P S$ quantum numbers):

$$
\begin{aligned}
& W_{L \mu}^{I}=\left(\bar{\psi}_{L} \gamma_{\mu} \frac{1}{2} \tau^{I_{\psi_{L}}}\right) \sim(3,1,1) \\
& W_{L_{\mu}}^{\prime}=\left(\bar{\psi}_{L^{\gamma}}^{\gamma} \psi_{L}\right) \quad \sim(1,1,1), \\
& w_{R \mu}^{I}=\left(\bar{\psi}_{R} \gamma_{\mu} \frac{1}{2} \tau{ }^{I} \psi_{R}\right) \sim(1,3,1), \\
& \mathrm{W}_{\mathrm{R} \mu}^{\prime}=\left(\bar{\psi}_{\mathrm{R}} \gamma_{\mu} \psi_{\mathrm{R}}\right) \sim(1,1,1), \\
& v_{\mu}^{A}=\left(s \stackrel{+\leftrightarrow}{D_{\mu}} \frac{1}{2} \lambda^{A} s\right) \sim(1,1,15), \\
& v_{\mu}^{\prime}=\left(s^{\dagger} D_{\mu} s\right) \sim(1,1,1), \\
& x^{A}=\left(s^{\dagger} \frac{1}{2} \lambda^{A} s\right) \sim(1,1,15), \\
& x^{I}=\left(s^{\dagger} s\right) \quad \sim(1,1,1) .
\end{aligned}
$$

Furthermore there will be bound states of higher spin and towers of excitations for all of the states of equs. (30) (33). The appearance of bound states in addition. to quarks, leptons and Higgs bosons is an unavoidable reature or preon models. Some of these new staes have an interesting phenomenology. The 15-plet $x^{A}$, for instance, contains scalars with the following colours and electric charges:

$$
\left\{x^{A}\right\}=\left\{\underline{8}_{0}, \underline{3}_{\frac{2}{3}}, \underline{3}_{-\frac{2}{3}}^{*} \underline{1}_{0}\right\} .
$$


The colour octet couples to quarks, the colour triplet to a quark-lepton pair (cf. Fig.2). It is the scalar leptoquark [2] which, as we will see in the following section, could cause rather spectacular effects.

In til pitecin nodel described duve lise strong and electroweak interactions are fundamental gauge interactions. There is an interesting alternative version [27] in which not the full SU(2) $\mathrm{L} \times \mathrm{U}(1)_{\mathrm{Y}}$ symmetry group is gauged but only the $U(1)$ subgroup generated by the electric charge. The weak interactions are mediated by the composite vector bosons $w_{L}$ and $w_{R}$ (cf. (33a), (33c)) and parity is assumed to be spontaneously broken, yielding $\mathrm{M}_{\mathrm{W}_{\mathrm{R}}}>\mathrm{M}_{\mathrm{W}_{\mathrm{T}}}$. The correct neutral current structure can be obtained through a dynamical $\mathrm{w}_{\mathrm{L}}^{3}-\mathrm{w}_{\mathrm{R}}^{3}-\gamma$ mixing. In a more radical approach even gluons and photon are considered as composite fields $[29,32]$.
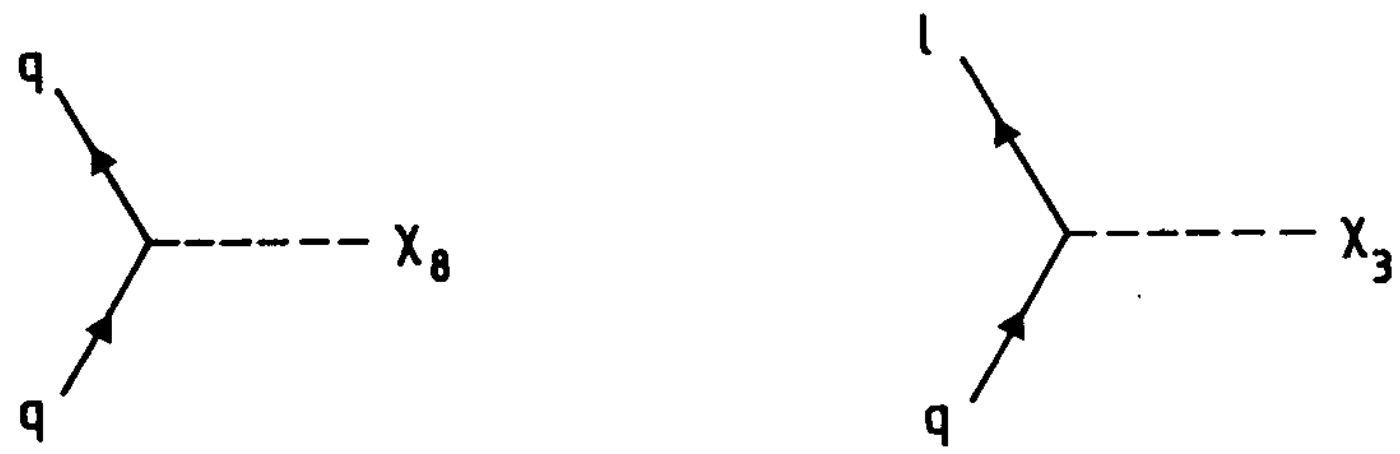

Fig. 2.: Couplings of colour octet and triplet (leptoquark) scalars to quarks and leptons. 
One of the main motivations for composite models is the replication of quark-lepton families. The bound states (30), however, represent only one family. Where do the others come from? Are they "radial excitations" or "pair excitations"? Should one introduce an additional preon carrying "generation number" or enlarge the global symmetry, for instance from $S U(4)$ to $S U\left(4 n_{f}\right)$ for $n_{f}$ generations? Is the family replication connected with the center $z_{n}$ of the hypercolour group $\mathrm{SU}(\mathrm{n})_{\mathrm{HC}}$ or enforced by anomaly matching? A number of interesting possibilities has been discussed in more detail by Harari [33], but a fully satisfactory solution of the generation puzzle still remains to be found.

What is the mass scale $\Lambda$ of the hypercolour interaction and the corresponding size $r \sim \frac{1}{\Lambda}$ of the composite quarks and leptons? As the preon theory has to explain the mass of the weak vector bosons (independent of whether they are elementary or composite) $\Lambda$, being the only mass scale of the theory, cannot be much larger than the Fermi scale $\mathrm{G}^{-1 / 2} \sim 300 \mathrm{GeV}$. As we will see in the following section such a "nearby compositeness" is barely compatible with experimental bounds on $\Lambda$.

So far we have only considered an example of the "quantum numerology" of preon models. The main dynamical problem of quark-lepton substructure, the appearance of almost massless composite fermions, will be discussed in $\sec .5$.

\section{BOUNDS ON THE SUBSTRUCTURE SCALE $\Lambda$}

The substructure scale of the preon model considered in the previous section was the Fermi scale, i.e., $\Lambda \sim G_{F}^{-1 / 2} \sim 300 \mathrm{GeV}$. There exists, however, at present no "standard composite model" and hence also no theoreti- 
cally preferred value of $\Lambda$. Indeed, preon models have been constructed with values of $\Lambda$ covering the whole range from $G_{F}^{-1 / 2}$ up to the Planck mass $M_{\mathrm{PJ}} \sim 10^{19} \mathrm{GeV}[18]$. In this section we will discuss the experimental bounds on $\Lambda$ which have been obtained from scattering experiments and rare nrocesses.

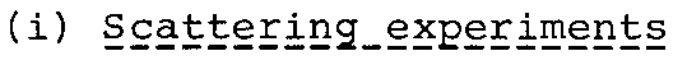

The classical process which probes the elementarity of the electron is Bhabba scattering (cf.Fig.3). A finite size of the electron would lead to an electromagnetic formfactor and a deviation from the pointlike coupling to the photon [34]:

$1 \rightarrow F\left(q^{2}\right)=1+\frac{q^{2}}{\Lambda_{F}^{2}}+o\left(\frac{1}{\Lambda_{F}^{4}}\right),\left|q^{2}\right|<\Lambda_{F}^{2}$.
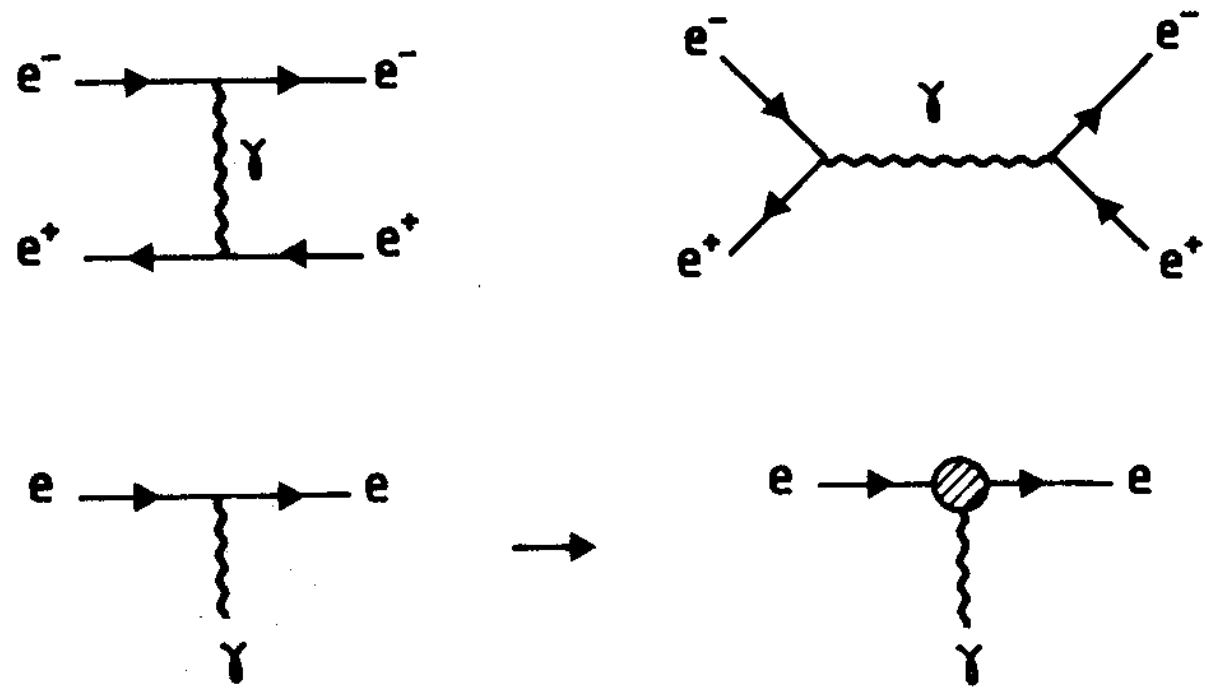

Fig. 3.: Bhabba scattering with formfactor modification. 
The current lower bound on the formfactor scale $\Lambda_{F}$ reads [34]

$\Lambda_{\mathrm{F}}>200 \mathrm{GeV}$,

which corresponds to the upper bound for the electron size

$r_{e} \approx \frac{1}{\Lambda_{F}}<10^{-16} \mathrm{~cm}$

If electrons are composite objects they will have contact interactions in addition to the standard model interactions. These contact interactions are caused by the interchange of constutuents or, equivalently, the exchange of heavy reaonances of various spins (cf. Fig.4). They are described by an effective Lagrangian of the form $\left(\left|q^{2}\right| \ll M_{v}^{2}, M_{s}^{2}\right)$

$L_{\text {eff }}=-\frac{g_{v}^{2}}{2 M_{v}^{2}} \bar{e} \gamma^{\mu} e \bar{e} \gamma_{\mu} e-\frac{g_{s}^{2}}{2 M_{s}^{2}} \bar{e} \bar{e} \bar{e}+\ldots$,

where $g_{v}, g_{S}, M_{v}$ and $M_{S}$ are the coupling constants and masses of a heavy vector $\mathrm{V}$ and a heavy scalar $\mathrm{S}$. For $\mathrm{a}$ strong binding force at the preon level one may expect, in analogy to $\mathrm{QCD}$, also large values for the coupling constants $a_{i}$ and $a_{0}$, i.e., $q_{v_{0}}^{2} / 4 \pi=0(1)$. It is therefore natural to define a substructure scale $\Lambda_{c}[35]$ as

$$
\frac{g^{2}}{M^{2}}=\frac{4 \pi}{\Lambda_{c}^{2}}
$$



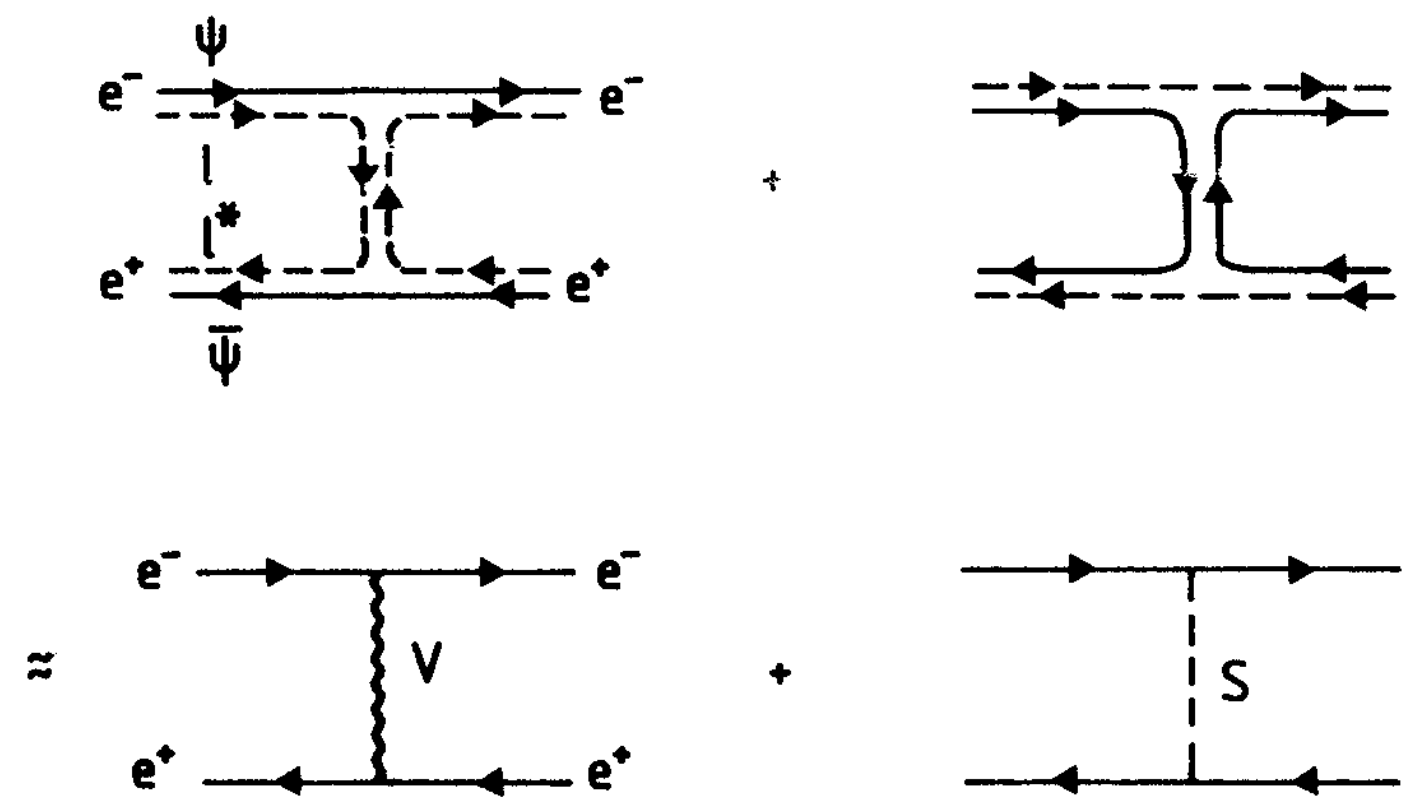

Fig. 4.: Dynamical origin of contact interactions: constituent exchange or, equivalently, exchange of heavy resonances.

The so defined scale $\Lambda_{c}$ corresponds physically to the mass of a strongly interacting resonance. Obviously, the formfactor correction (35) also leads to a contact texm. From

$2 \frac{e^{2}}{q^{2}} \frac{q^{2}}{\Lambda_{F}^{2}}=\frac{4 \pi}{\Lambda_{c}^{2}}$

one obtains for the relation between the two scales $\Lambda_{F}$ and $\Lambda_{C}$ :

$\Lambda_{\mathrm{C}}=\frac{1}{\sqrt{2 \alpha}} \Lambda_{\mathrm{F}} \approx 8 \Lambda_{\mathrm{F}}$ 
Contact interactions may have various Lorentz structures. of particular interest are the chirality conserning leftleft, right-right, vector-vector and axial vector-axial vector interactions (LL-, RR-, VV- and AA-"models"). Eichten, Lane and Peskin have studied the effect of the contact interactions on the angular distribution in Bhabba scattering (cf. Fig.5). Their analysis of PETRA data at $\sqrt{\mathrm{s}}=35 \mathrm{GeV}$ led to the bounds [35]

$\Lambda_{C}> \begin{cases}0.75 \mathrm{TeV}, & \text { for LL-, RR-models } \\ 1.5 \mathrm{TeV}, & \text { for } \mathrm{VV}-, \text { AA-models }\end{cases}$

The analysis of Bhabba scattering at $\sqrt{\mathrm{s}}=100 \mathrm{GeV}$ at SLC and LEP (cf. Fig.6) is expected to yield the bounds [35]

$\Lambda> \begin{cases}2 \mathrm{TeV}, & \text { for LL-, RR-models } \\ 5 \mathrm{TeV}, & \text { for } \mathrm{VV}-, \text { AA-models . }\end{cases}$

Rückl has studied the effect of contact interactions in ep-scattering $[37,38]$. For the LL-model

$$
L_{e f f}=\frac{4_{I I}}{\Lambda_{C}} \vec{e}_{L} \gamma^{\mu_{e}} \bar{\tau}_{L}{ }_{L} \gamma_{\mu} q_{L}
$$

he finds that the structure function $F_{2}$ at $x=0.5$ and $Q^{2}=(100 \mathrm{GeV})^{2}$ charges by $25 \%(60 \%)$ for $\Lambda_{\mathrm{C}}=2 \mathrm{TeV}$ (1.5 TeV) (cf. Fig.7), which illustrates the sensitivity of HERA experiment to substructure effects. 

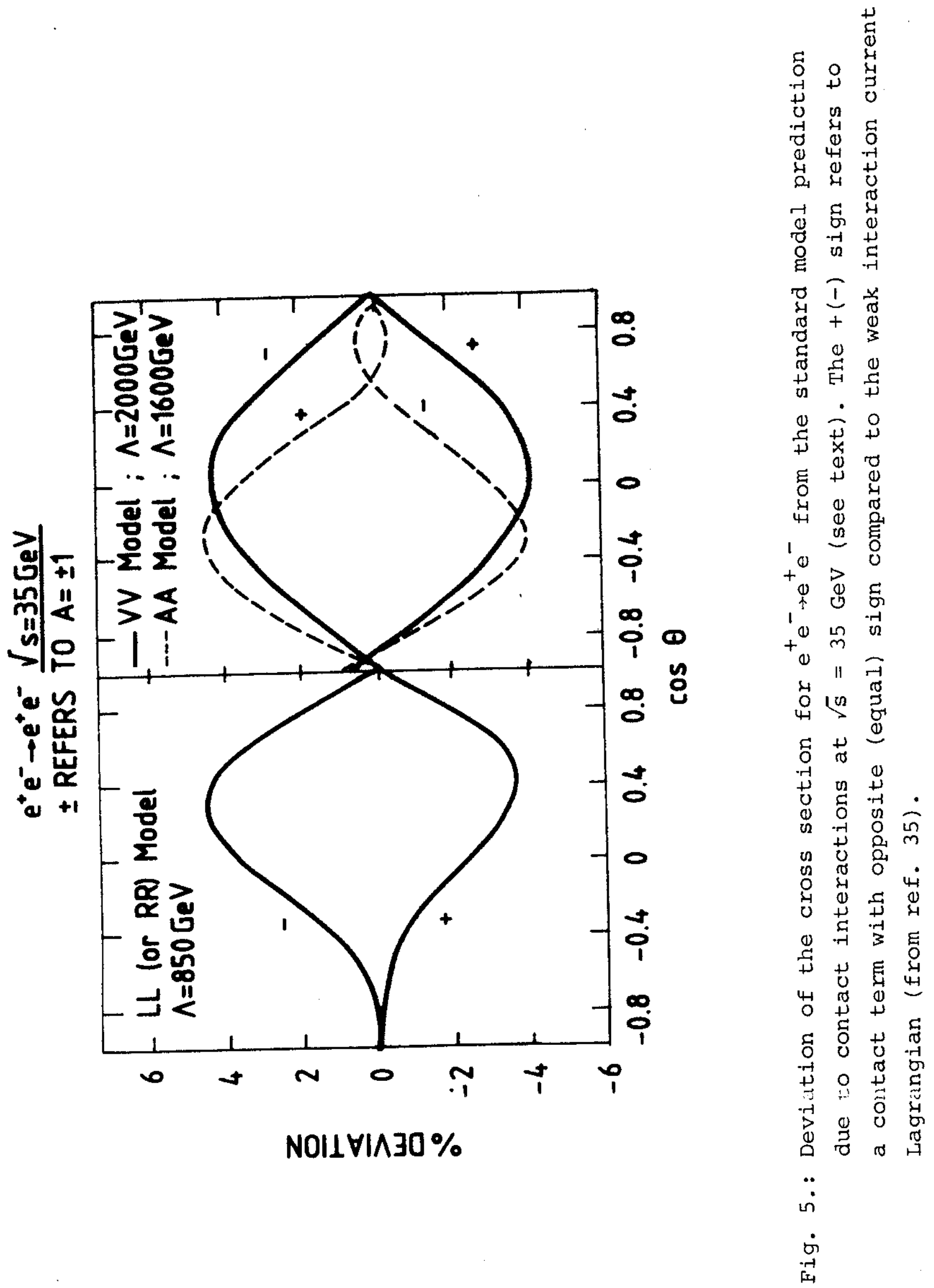


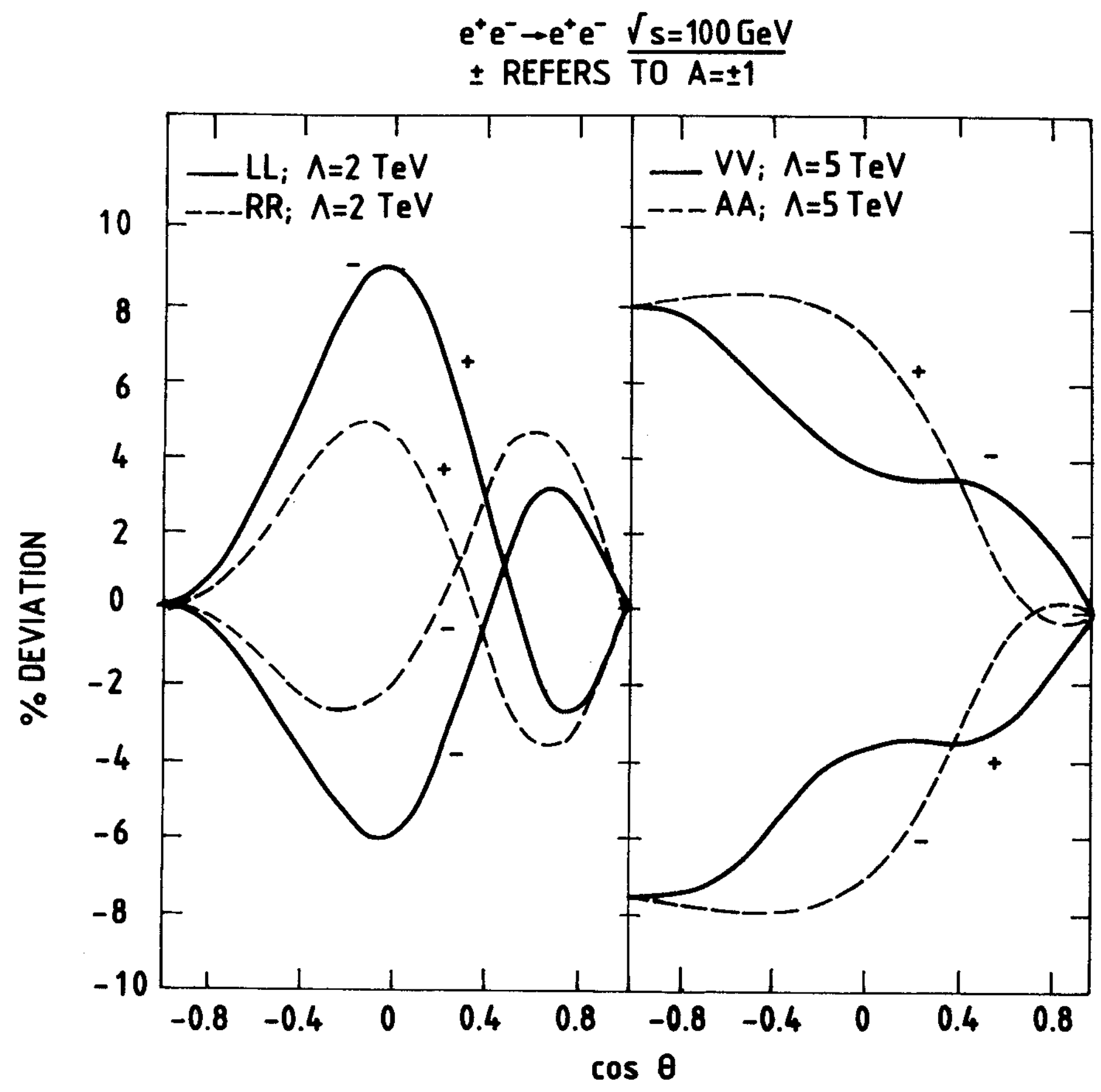

Fig. 6.: Deviation of the cross section for $e^{+} e^{-} \rightarrow e^{+} e^{-}$ from the standard model prediction due to contact interactions at $\sqrt{\mathrm{s}}=100 \mathrm{GeV}$. (cf. Fig.5, from ref.35). 


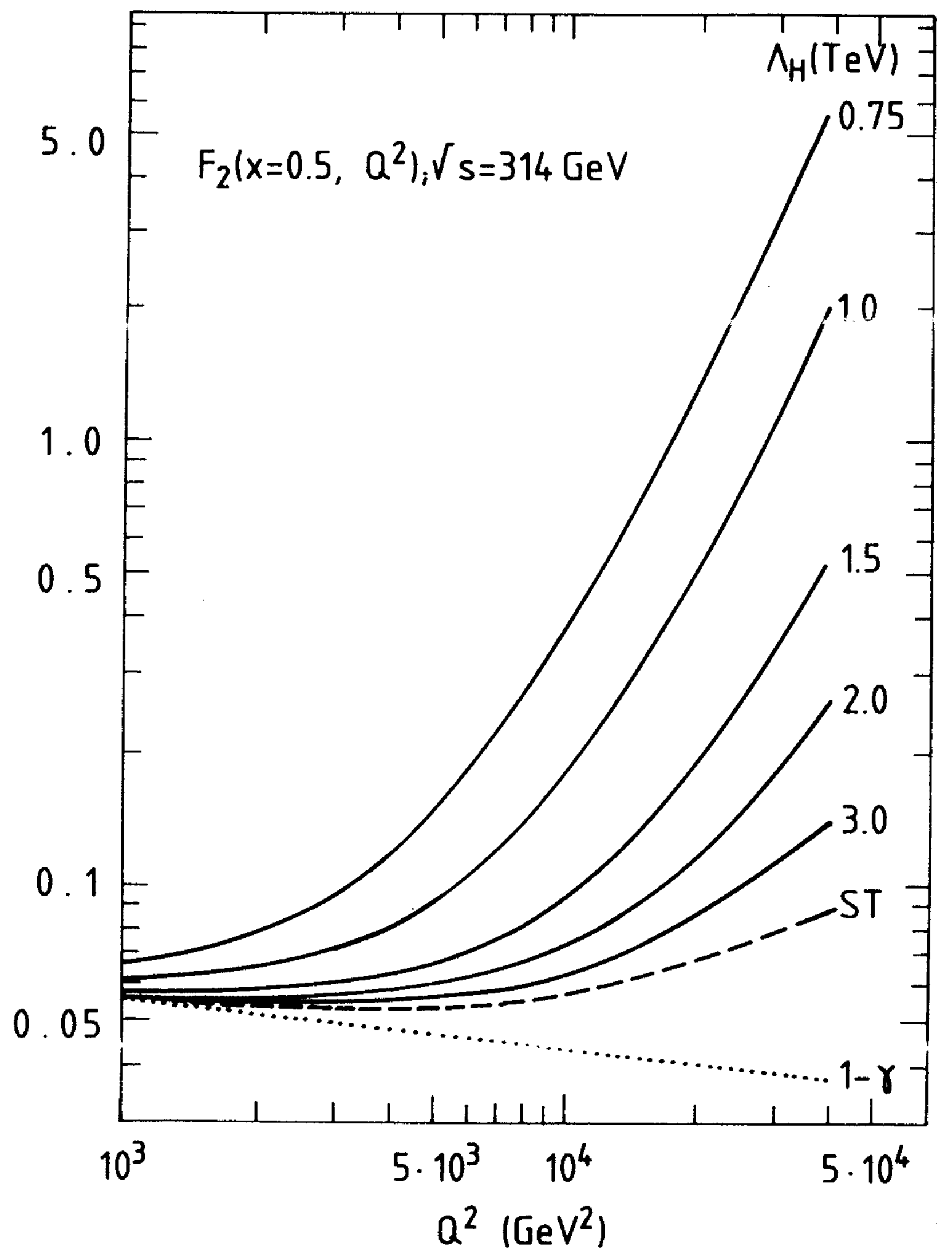

Fig. 7.: Deviation of the proton structure function $F_{2}$ at $\mathrm{x}=0.5, \sqrt{\mathrm{s}}=314 \mathrm{GeV}$, from the standard model prediction due to the contact interaction (43) (from ref. 37). 
Following Abolins et al. [36] we have listed in table II the bounds on the substructure scale $\Lambda_{C}$ and the (electromagnetic or colour) formfactor scales $\Lambda_{F}$ for various processes which can be obtained at the next generation of accelerators. Despite the considerable increase in energy the bounds will be improved by at most a factor of three compared to the current bound (41).

Table II: Expected bounds from future colliders on the mass scales $\Lambda_{C}$ and $\Lambda_{F}$ for various processes. In the second column the assumed integrated luminosity per year is listed (from ref.36). The values for $\Lambda_{F}$ and estimated according to equ. (40) with $\alpha_{E M}=0.008$ and $\alpha_{S}=0.1$.

\begin{tabular}{|c|c|c|c|c|}
\hline $\begin{array}{l}\text { process } \\
\sqrt{\mathrm{s}}[\mathrm{GeV}]\end{array}$ & $\int L d t / y r$ & $\begin{array}{c}\Lambda_{\mathrm{C}} \\
{[\mathrm{TeV}]}\end{array}$ & $\begin{array}{c}\Lambda_{\mathrm{F}} \\
{[\mathrm{GeV}]}\end{array}$ & $\begin{array}{c}\text { subprocess } \\
\text { tested }\end{array}$ \\
\hline ep, $10 \times 1000$ & $10^{39}$ & 4 & 500 & eq $\rightarrow$ eq \\
\hline$e^{+} e^{-}, \leq 100$ & $10^{38}$ & 2 & 250 & $e^{+} e^{-} \rightarrow e^{+} e^{-}$ \\
\hline$e^{+} e^{-}, \geq 200$ & $10^{38}$ & 3 & 380 & \\
\hline$p p, 2000$ & $10^{40}$ & 2 & 890 & $q q \rightarrow q g$ \\
\hline$p \bar{p}, 2000$ & $10^{37}$ & 1.5 & 700 & D. \\
\hline $\mathrm{p} \overline{\mathrm{p}}, 2000$ & $10^{37}$ & 4 & 500 & $q \vec{q} \rightarrow \mu^{+} \mu^{-}$ \\
\hline
\end{tabular}




\section{(ii) Rare_processes}

Rare processes provide stringent constraints on possible extensions of the standard model. They have been studied in connection with horizontal interactions $[39,40]$, extended technicolour theories $[41,42]$ as well as composite models [43-46]. A systematic study of possible manifestations of physics beyond the standard model can be carried out by examining all higher dimensional operators [44] which can be constructed from the fields of the standard model. In preon models such operators, which contain the contact interactions, will be generated through the exchange of heavy resonances. There exist more than 100 operators of dimension $6[44,46]$. Some interesting examples are:

$$
\begin{aligned}
& o_{1}=\frac{1}{2} g_{2} \stackrel{1}{1 j}^{\dot{j}} \quad \bar{\ell}_{L i} \sigma^{\mu \nu}{ }_{\tau}{ }_{\phi} e_{R} j_{W \nu}^{I}+c . c ., \\
& w_{\mu \nu}^{I}=\partial_{\mu} w_{\nu}^{I}-\partial_{\nu} W_{\mu}^{I}+g_{2} \varepsilon_{I J K} W_{\mu}^{I} w_{\nu}^{K} \text {, } \\
& o_{2}=\frac{1}{2} g_{1} \Gamma_{2 j}^{j} \bar{l}_{L i} \sigma^{\mu \nu} \phi e_{R}^{j} B_{\mu \nu}+c . c ., \\
& \mathrm{B}_{\mu \nu}=\partial_{\mu} \mathrm{B}_{\nu}-\partial_{\nu} \mathrm{B}_{\mu}, \\
& O_{3}=\Gamma_{3 j}^{i} \phi^{\dagger} \phi \bar{l}_{L i} \phi e_{R}^{j}+\text { c.c. , } \\
& o_{4}=\Gamma_{4 k 1}^{i j} q_{L i} \gamma^{\mu} q_{L}^{k} \bar{l}_{L j} \gamma_{\mu} \ell_{L}^{\ell},
\end{aligned}
$$

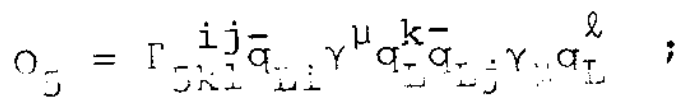

the matrices $\Gamma_{1 j}^{i}, \ldots, \Gamma_{5 \mathrm{k} l}^{i j}$ contain a priori arbitrary flavour mixing .

The flavour diagonal part of the operators $\mathrm{O}_{1}$ and $\mathrm{O}_{2}$ contributes, after spontaneous symmetry breaking, to the anomalous magnetic moments of the leptons. Following the 
convention of the contact interactions in defining a substructure scale, one obtains (cf. Fig.8)

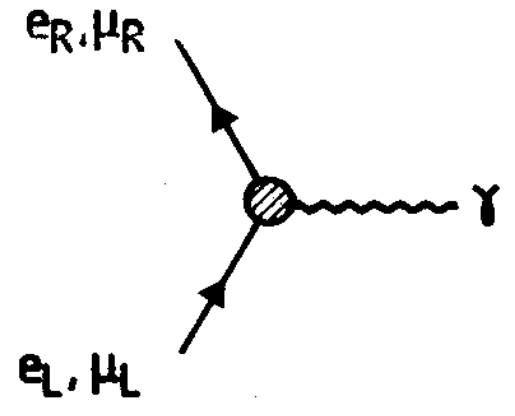

a)

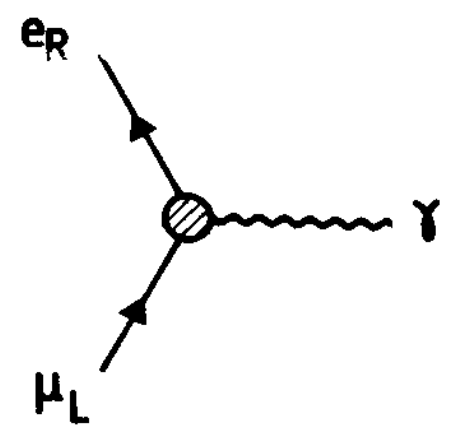

b)

Fig. 8.: Magnetic dipole couplings of leptons causing anomalous magnetic moments (a) and the $\Delta G=1$ transition $\mu \rightarrow$ er (b).

$o_{a}=e \frac{4 \pi}{\Lambda^{2}} v \bar{l}_{L i} \bar{\sigma}^{\mu \nu} e_{R}^{i} F_{\mu \nu}+$ c.c.,

where $F_{\mu \nu}$ is the electromagnetic field strength tensor and $v$ the vacuum expectation value of the Higgs field. Recalling the Gordon decomposition

$$
\begin{aligned}
\bar{\psi}_{\mu} \psi A^{\mu} & =\frac{1}{2 m} \bar{\psi}^{-i_{D}} \psi_{A} A^{\mu}+\frac{1}{4 m} \bar{\psi} \bar{\sigma}^{\mu \nu} \psi \mathrm{F}_{\mu \nu}+\partial_{\mu}(\ldots), \\
D_{\mu} & =\partial_{\mu}+\text { ie } A_{\mu},
\end{aligned}
$$

one immediately obtains for the anomalous magnetic moment corresponding to (45):

$$
\delta a_{\ell}=16 \frac{m_{\ell} v}{\Lambda^{2}} .
$$


Usually anomalous magnetic moments due to compositeness $[47,48]$ are estimated as

$$
\delta a_{\ell}^{\prime}=\left(\frac{m_{\ell}}{\Lambda}\right)^{2},
$$

which leads to weaker bounds on $\Lambda$ than (47). In order to obtain (48) from the operators $O_{1}$ and $O_{2}$ one has to assume that the matrices $\Gamma_{1,2}$ are proportional to the Yukawa coupling matrix $g(e)^{1}$. For arbitrary matrices $\Gamma$ a stringent bound follows already from $\mathrm{O}_{3}$ which, after spontaneous symmetry breaking, leads to the mass term

$$
O_{m}=\frac{4 \pi}{\Lambda^{2}} v^{3}\left(\bar{l}_{L i} e_{R}^{i}+\bar{e}_{R i}^{\ell}{ }^{i}\right)
$$

Requiring the corresponding lepton mass to be smaller than the electron mass one finds

$$
\Lambda>360 \mathrm{TeV} \text {. }
$$

Agreement between QED calculations and experiment allows an anomalous magnetic moment $|\delta a|<2 \times 10^{-10}\left(3 \times 10^{-8}\right)$ [49] for the electron (muon). Using equ. (47) ((48)) this implies the bounds on $\Lambda$

$$
\Lambda> \begin{cases}150 \mathrm{TeV} & (40 \mathrm{GeV}), \text { electron } \\ 170 \mathrm{TeV} & (580 \mathrm{GeV}), \text { muon } .\end{cases}
$$

Bounds much stronger than (50) or (51) can be obtained from flavour-changing processes. It is convenient to classify them by means of a generation number [40]. Transitions between the $e(\mu)$ - and $\mu(\tau)$-families are $\Delta G=1$ processes, transitions between the $e$ - and $\tau$-families $\Delta G=2$ processes. 
The flavour off-diagonal part of the operators $o_{1}$ and $\mathrm{O}_{2}$ contains a term causing the $\Delta \mathrm{G}=1$ process $\mu \rightarrow \mathrm{er}$ (cf.Fig. 8) :

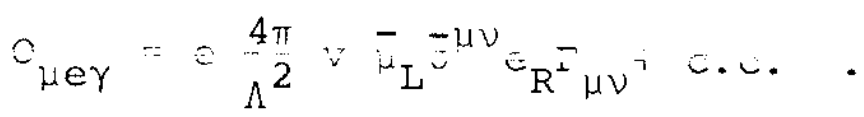

From the experimental upper bound for the branching ratio,

$B R(\mu \rightarrow e \gamma)<1.7 \times 10^{-10}[50]$, one obtains

$\Lambda>3 \times 10^{4} \mathrm{TeV}(200 \mathrm{TeV})$,

where the number in bracket has been obtained by replacing in (52) $4 \pi v$ by $m_{\mu}$.

A paticularly dangerous process for preon models of Pati-Salam type is the decay $\overline{\mathrm{K}}^{\circ} \rightarrow \mu^{-} \mathrm{e}^{+}$(cf. Fig.9).
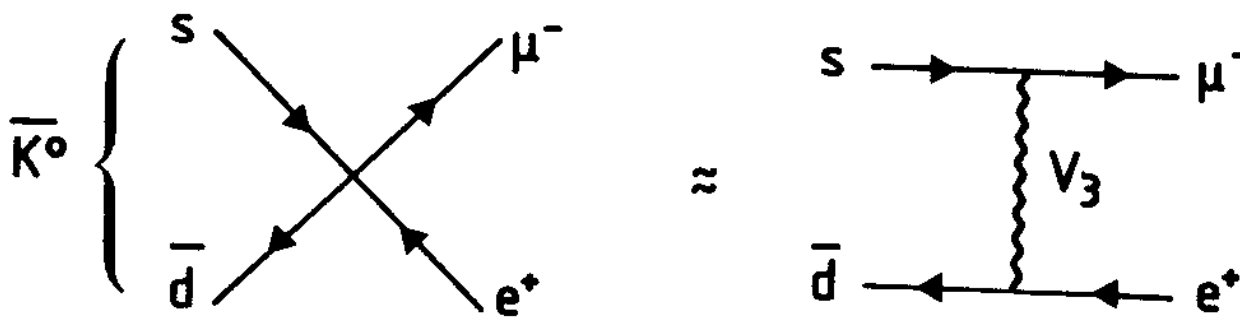

$(\Delta G=0)^{2}$

Fig. 9.: The $\Delta \mathrm{G}=\mathrm{O}$ contact interaction for $\overline{\mathrm{K}}^{\circ} \rightarrow \mu^{-} \mathrm{e}^{+}$induced by the exchange of a leptoquark vector boson.

The corresponding operator $\mathrm{O}_{4}$ can be generated through the exchange of a leptoquark $V_{3}$ with two $\Delta G=0$ vertices. From 
$O_{K \circ \mu e}=\frac{4 \pi}{\Lambda} \bar{d}_{L} \gamma^{\mu} s_{L} \bar{\mu}_{L} \gamma_{\mu} e_{L}+c . c$.

and the bound on the ratios of partial widths (cf.[40],[50])

$\frac{\Gamma\left(K_{L}^{O} \rightarrow \mu^{ \pm} e^{\tau}\right)}{\Gamma\left(K^{+} \rightarrow \mu^{+} \nu_{\mu}\right)}<2.3 \times 10^{-6}$

one obtains

$\Lambda>110 \mathrm{TeV}$.

The strongest bound follows from the $\Delta G=2$ process of $\mathrm{K}^{\circ}-\overline{\mathrm{K}}^{\circ}$ mixing. Comparing the contribution of the operator $\mathrm{O}_{5}$ (cf. Fig.10),

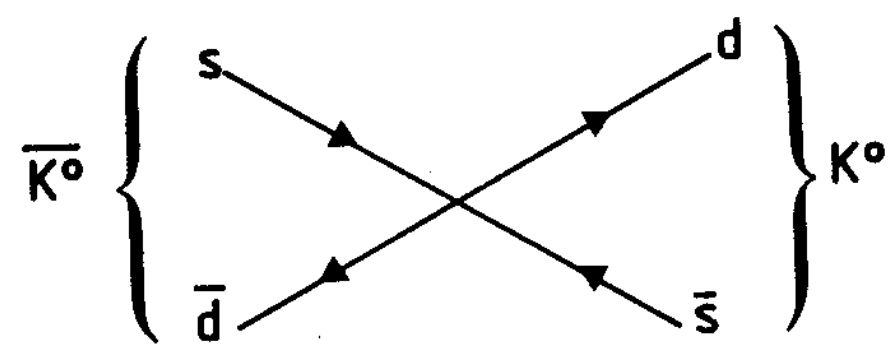

Fig. 10.: The contact interaction for $\mathrm{K}^{\circ}-\overline{\mathrm{K}}^{\circ}$ mixing.

$o_{K O} \bar{K}^{\circ}=\frac{2 \pi}{\Lambda} \bar{s}_{L} \gamma^{\mu} d_{L} \bar{s}_{L} \gamma_{\mu} d_{L}+c . c$.

with the standard model box graph contribution of Gaillard and Lee one finds (cf. [40])

$\Lambda>4300 \mathrm{TeV}$. 
Clearly the bounds (53), (56) and (58) are incompatible with "nearby compositeness", i.e., $\Lambda<1 \mathrm{TeV}$. It is conceivable however, that the flavour changing processes are torbidden by appropriate symmetries. Electron or muon number conservation, for instance, which are symmetries of the standard model without right-handed neutrinos, would forbid the decay $\mathrm{K}_{\mathrm{L}}^{\circ} \rightarrow \mu^{ \pm} \mathrm{e}^{\overline{+}}$. An alternative dynamical mechanism to prevent this decay has been suggested by Greenberg, Mohapatra and Nussinov [51]. As can be seen from Fig.11, the process $\overline{\mathrm{K}}^{\circ} \rightarrow \mu^{-} \mathrm{e}^{+}$is not allowed, if a generation quantum is associated with the scalar preons, i.e., instead of 4 preons $x=\left(h^{\alpha}, l\right)$ one introduces $4 n_{f}$

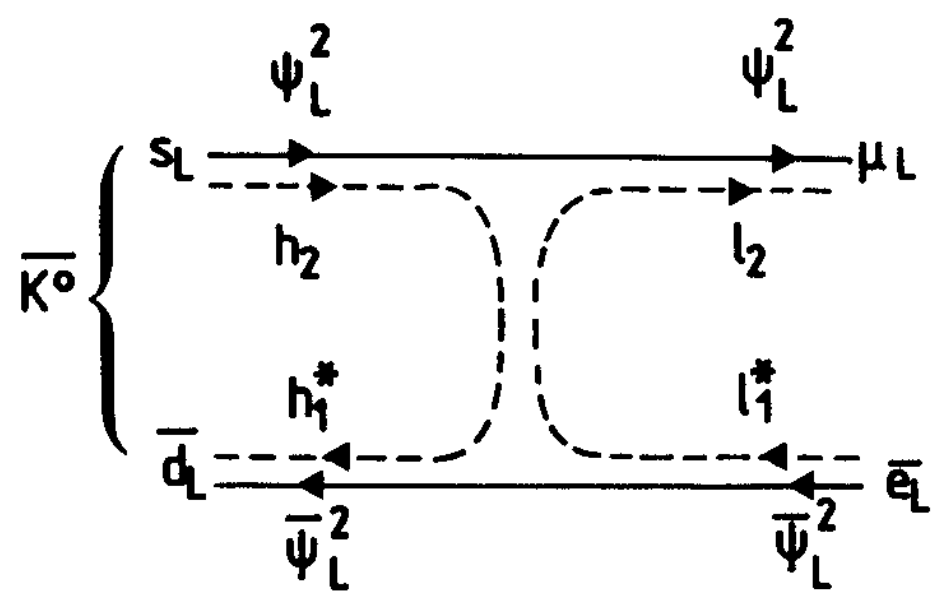

Fig. 11.: Preon diagram for the decay $\overline{\mathrm{K}}^{\circ} \rightarrow \mu^{-} \mathrm{e}^{+}$. For unbroken generation symmetry the scalar lines cannot be connected and the process is forbidden (cf. ref.51).

preons $x_{i}=\left(x_{i}^{\alpha}, l_{i}\right), i=1 \ldots n_{f}$. This, however, shifts the problem of family replication to the preon level which is contrary to the motivation for quark-lepton substructure.

A more detailed discussion of dynamical mechanisms 
which can guarantee "natural flavour conservation" will be given in ref.46. The only unavoidable low-energy effects of compositeness appear to be anomalous magnetic moments of quarks and leptons, which, according to (51), are marginally consistent with a substructure scale below $1 \mathrm{TeV}$.

\section{COMPOSITE W-BOSONS}

In preon models with "nearby compositeness", i.e., $\Lambda \sim G_{F}^{-1 / 2} \sim 300 \mathrm{GeV}$, the weak intermediate vectors bosons appear generally as bound states and not as fundamental gauge bosons. The idea of composite $W$-bosons is compatible with all present experimental results and represents a consistent alternative to the gauge theory of weak interactions. The main ingredients in models with composite W-bosons are the dynamical mixing of the photon with the neutral W-boson, first considered by Bjorken [52] and Hung and Sakurai [53] in connection with the neutral current structure of weak interactions, and the principle of vector meson dominance, as discussed by Fritzsch, Kögerler and Schildknecht $[54,55]$. This picture of composite $w$-bosons appears to be dynamically realized in theories with scalar condensates [56-59]. The effective Lagrangian of composite $W$-bosons is that of a massive Yang-Mills theory [60].

The effective Lagrangian of a preon theory with composite $W$-boson contains kinetic terms for the $\mathrm{SU}(2) \mathrm{tri-}$ plet of $W$-bosons and the photon, the mass term for the $W$-bosons and the couplings of the vector bosons to the conserved SU(2) and electromagnetic currents:

$$
L=-\frac{1}{4} W_{\mu \nu}^{I} W^{I \mu \nu}-\frac{1}{4} F_{\mu \nu} F^{\mu \nu}+\frac{1}{2} M_{W}^{2} W_{\mu}^{I} W^{I \mu}-
$$




$$
-g W^{I \mu} j_{\mu}^{I}-e A^{\mu} j_{\mu}^{E M}
$$

with

$$
\begin{aligned}
& W_{\mu \nu}^{I}=\partial_{\mu} W_{\nu}^{I}-\partial_{\nu} W_{\mu}^{I}+g \varepsilon_{I J K} W_{\mu}^{J} W_{\nu}^{K}, \\
& F_{\mu \nu}=\partial_{\mu} A_{\nu}-\partial_{\nu} A_{\mu}, \quad \partial^{\mu} j_{\mu}^{I}=\partial^{\mu}{ }_{j}^{E M}=0 .
\end{aligned}
$$

The dynamical mixing of the elementary photon with the composite neutral $\mathrm{W}^{3}$-boson is described by the $\mathrm{U}(1)_{\mathrm{EM}}$ gauge invariant "current mixing" [61]

$$
L_{\gamma W}=-\frac{1}{2} \lambda F_{\mu \nu} W^{3 \mu \nu}=\lambda A_{\nu} \square W^{3 \nu}+\partial_{\mu}(\ldots)
$$

From equs. (59) and (60) one reads off the propagators and vertices shown in Fig. 12 .

$$
\text { Using the Feynman rules of Fig.12 one can easily }
$$
construct the effective current - current Lagrangian as a power series in $\lambda$ :

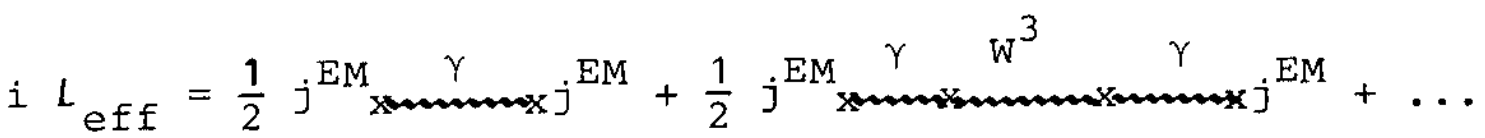

$$
\begin{aligned}
& \left(-\lambda q^{2}\right)^{2}
\end{aligned}
$$

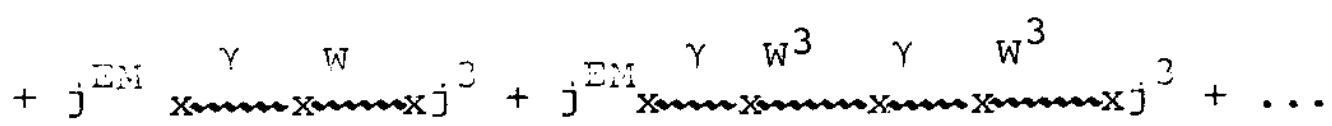

$$
\begin{aligned}
& -\lambda q^{2} \quad\left(-\lambda q^{2}\right)^{3}
\end{aligned}
$$

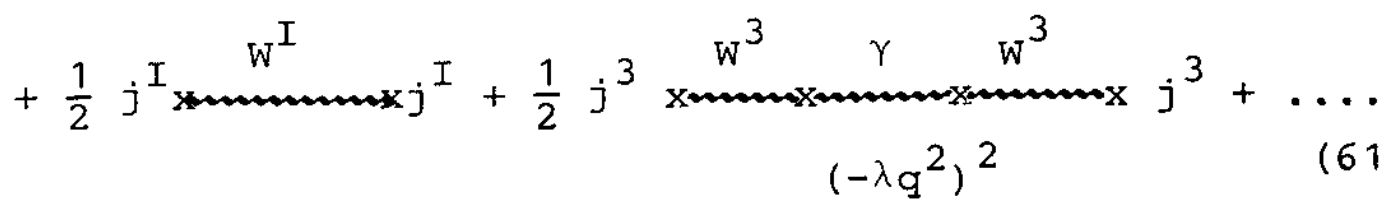




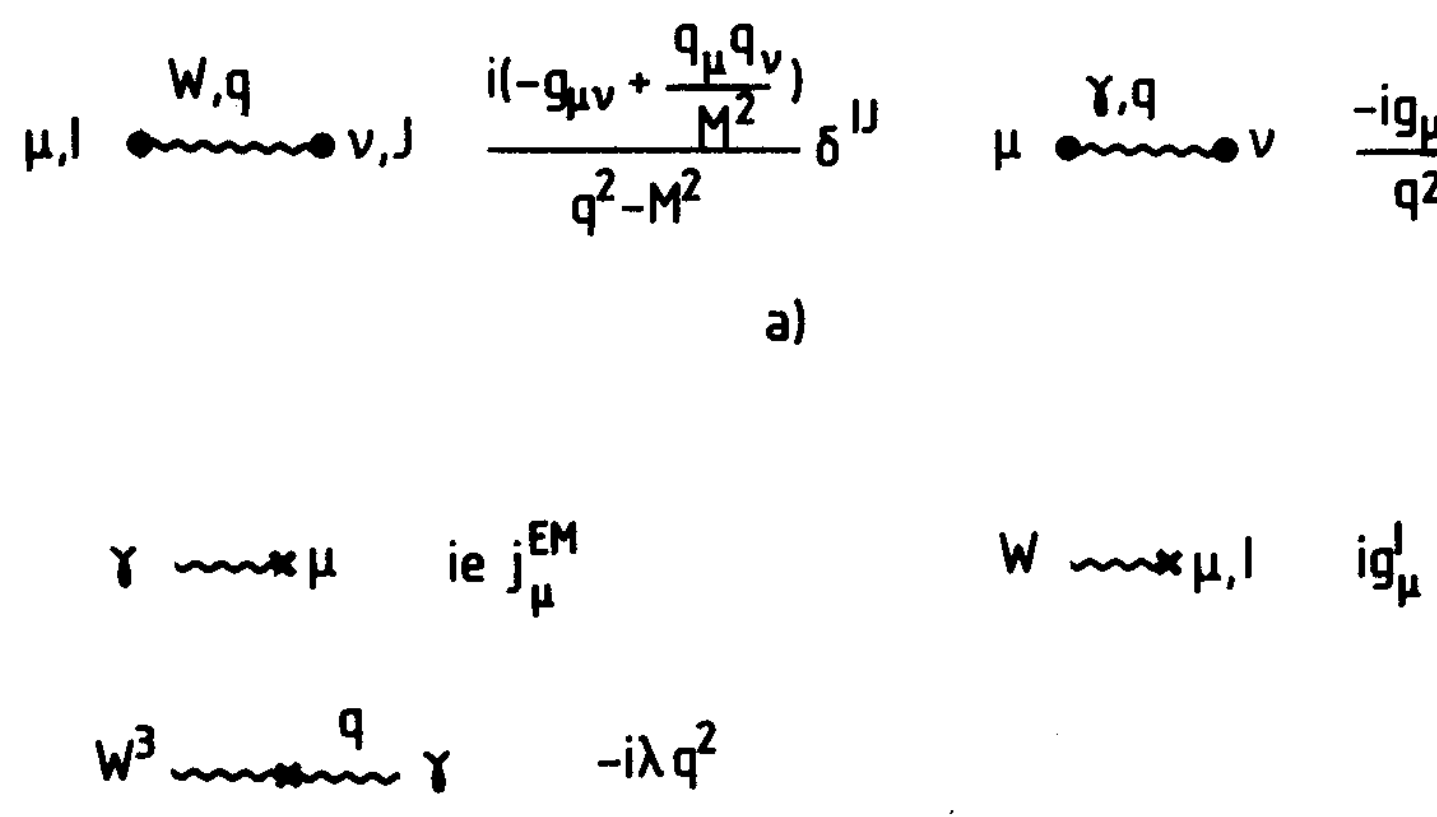

b)

Fig. 12.: Propagators (a) and vertices (b) from the Lagrangian (59).

For $\left|\mathrm{q}^{2}\right| \ll \mathrm{M}_{\mathrm{W}}^{2}$ only four terms remain:

$$
\begin{aligned}
& L_{\text {eff }} \underset{\left|q^{2}\right|<M_{W}^{2}}{\approx} \frac{1}{2}\left(\frac{e^{2}}{q^{2}}-\frac{e^{2} \lambda{ }^{2}}{M_{W}^{2}}\right) j_{\mu}^{E M_{j} E M \mu}-\frac{1}{2} \frac{q^{2}}{M_{W}^{2}} j_{\mu}^{I} I \mu \\
& +\frac{\mathrm{e} \lambda \mathrm{g}}{\mathrm{M}_{W}^{2}} j_{\mu}^{\mathrm{EM}} j^{3 \mu} \\
& =\frac{1}{2} \frac{e^{2}}{q^{2}} j_{\mu}^{E M} j^{E M \mu}-\frac{1}{2} \frac{q^{2}}{M_{W}^{2}} j_{\mu}^{+} j^{-\mu} \\
& -\frac{1}{2} \frac{g^{2}}{M_{W}^{2}}\left(j_{\mu}^{E M}-\frac{e \lambda}{g} j_{\mu}^{3}\right)^{2},
\end{aligned}
$$


where

$j_{\mu}^{ \pm}=\sum_{i} \bar{f}_{L i} \gamma_{\mu} \tau^{ \pm} f_{L i}$

The result (62) is precisely the standard model weak interaction Lagrangian which, as we have seen, follows simply from global SU(2) invariance and current mixing. The Weinberg angle is a function of $e, g$ and $\lambda$ :

$\sin ^{2} \theta_{W}=\frac{e^{\lambda}}{g}$

The $\gamma-w^{3}$ mixing also causes a mass shift of the neutral W-boson. The kinetic terms of (59) and (60) correspond to the propagator matrix

$\Delta^{-1}\left(q^{2}\right)=\left(\begin{array}{cc}q^{2} & \lambda q^{2} \\ \lambda q^{2} & q^{2}-M_{W}^{2}\end{array}\right)$,

whose determinant,

$\operatorname{det} \Delta^{-1}\left(q^{2}\right)=q^{2}\left[q^{2}\left(1-\lambda^{2}\right)-M_{W}^{2}\right]$,

has the eigenvalues $\mathrm{q}^{2}=0$ and $\mathrm{q}^{2}=\mathrm{M}_{\mathrm{W}}^{2} /\left(1-\lambda^{2}\right)$ which correspond to the massless photon and the massive $z$-boson with

$M_{Z}=\frac{M_{W}}{\sqrt{1-\lambda^{2}}}$.

From equ. (63), (66) and $\frac{\mathrm{g}^{2}}{\mathrm{M}^{2}}=4 \sqrt{2} \mathrm{G}_{\mathrm{F}} \mathrm{M}_{\mathrm{Z}}$ can be determined as function of $\mathrm{M}_{\mathrm{W}}$ (cf. Fig.13). Neinberg's mass prediction is obtained, if one supplements (63) by the so called 


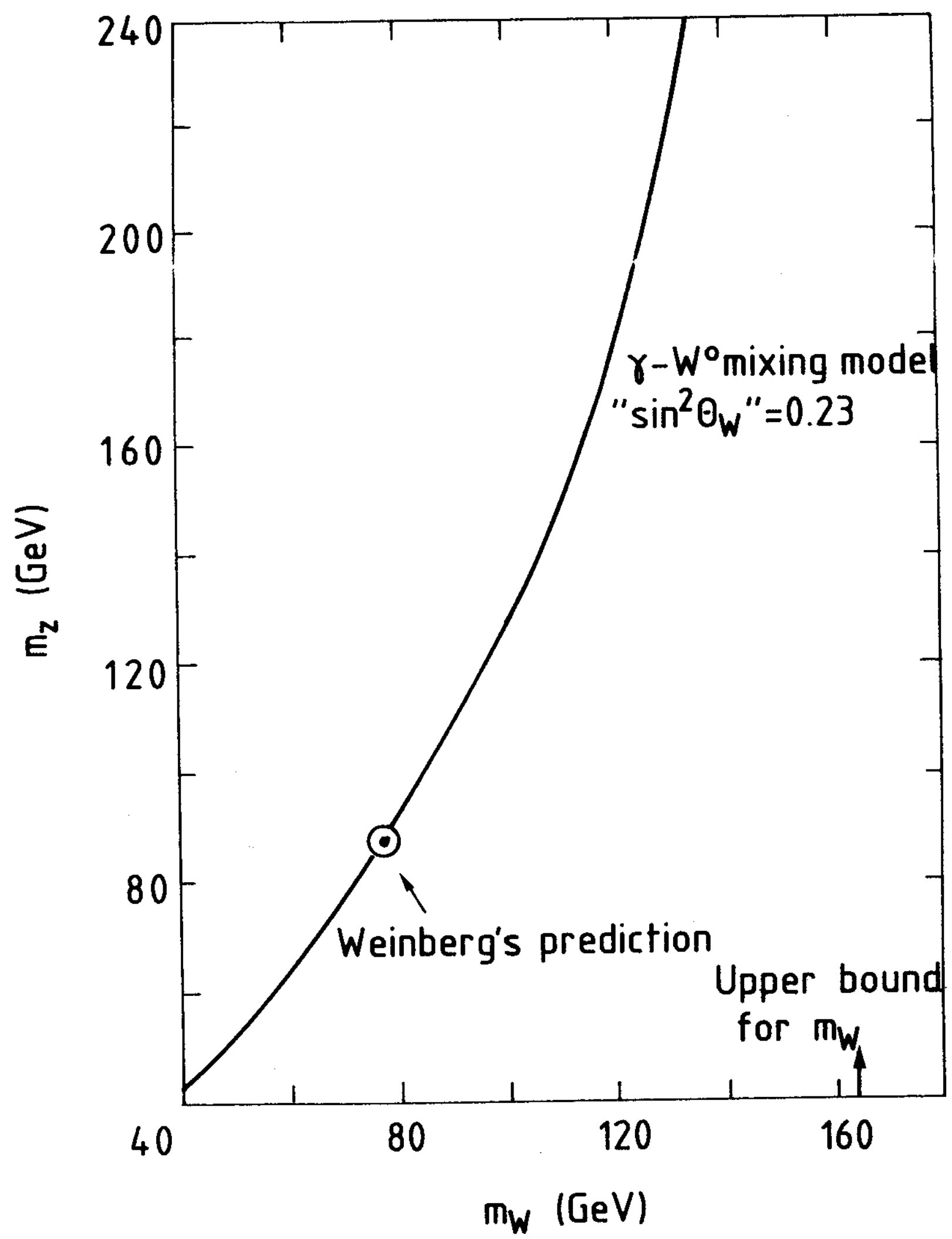

Fig. 13.: $M_{Z}$ as function of $M_{W}$ in the $W^{3}-\gamma$ mixing scheme (from ref.62). 
"unification condition" [53]

$\mathrm{e}=\mathrm{g} \lambda$,

which implies

$\lambda=\sin \theta_{W^{\prime}} \quad M_{Z}=\frac{M_{W}}{\cos \theta_{W}}$.

One easily verifies that, if the "unification condition" holds, the effective current-current Lagrangian becomes $S U(2)$ invariant at large momentum transfers. Contrary to (61) one has to sum up a geometric series in $\lambda$ for $q^{2}>M_{W}^{2}$, yielding the result $(e=g \lambda)$

$$
\begin{aligned}
& L \operatorname{eff}{\left|q^{2}\right|>>M_{W}^{2}}_{1-\lambda^{2}}^{\approx} \frac{1}{q^{2}}\left\{\frac{1}{2} e^{2} j_{\mu}^{E M}{ }^{E M \mu}-\lambda \text { eg } j_{\mu}^{E M} j^{3 \mu}\right. \\
& \left.+\frac{1}{2} g^{2}\left(1-\lambda^{2}\right) j_{\mu}^{I} j^{I \mu}+\frac{1}{2} g^{2} \lambda^{2} j_{\mu}^{3} j^{3 \mu}\right\} \\
& =\frac{1}{2 q^{2}}\left\{g^{\prime 2} j_{\mu}^{Y} j^{Y \mu}+g^{2} j_{\mu}^{I} j^{I \mu}\right\},
\end{aligned}
$$

where $j_{\mu}^{Y}$ and $g^{\prime}$ are the standard model hypercharge current and coupling constant,

$$
i_{\mu}^{Y}=i_{\mu}^{E M}-i_{\mu}^{3} ; \quad \sigma^{\prime}=\frac{e}{\sqrt{1-\lambda^{2}}}=\frac{e}{\cos \theta}
$$

The Lagrangian (69) corresponds to the exchange of massless $B-$ and $W$-bosons as in the standard model for large-momentum transfers.

The "unification condition" (67) can also be understood as a consequence of vector meson dominance $[54,55]$. The experi- 
mental value of $\sin ^{2} \theta_{W} \approx 0.23$ requires a large $\gamma-W^{3}$ mixing parameter $\lambda$, whereas in analogy to the $\rho-\gamma$ mixing in hadron physics one would expect $\lambda=O\left(\alpha_{E M}\right)$. The required large dynamical $\gamma-W^{3}$ mixing can be understood, if the exoitation enexgy of the compusite $w$-bosons is of the oraer of $1 \mathrm{TeV}$, i.e., much larger than the mass of the lowest vector boson $[63,64]$ (cf. Fig.14). In this case, however,

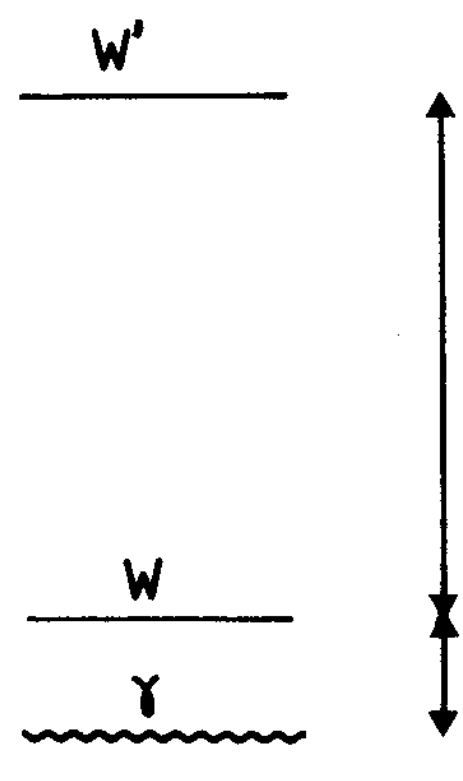

$\sim 1 \mathrm{TeV}$

\section{$\sim 100 \mathrm{GeV}$}

Fig. 14.: Pattern of vector boson masses required for a large dynamical $W^{3}-\gamma$ mixing.

one expects $W$-dominance $[54,55]$, i.e., the formfactor of the isovector part of the electromagnetic current should be dominated by the isovector boson of lowest mass (cf. Fig.15). This implies precisely the "unification condition" (67).

The extension of vector meson dominance beyond the isovector part of the electromagnetic current requires at least one additional $\mathrm{SU}(2)$ singlet vector boson $V$, which corresponds to the B-boson of the standard model. 


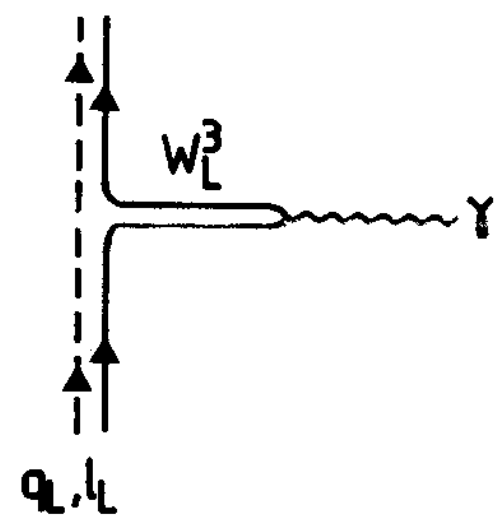

Fig. 15.: W-dominance of the isovector part of the electromagnetic form factor.

In Pati-Salam type preon models the required isosinglet $\mathrm{V}$ is part of a SU(4) PS 15-plet which contains in addition leptoquarks $\mathrm{V}_{3}$ and $\mathrm{V}_{3}$. and a colour octet vector boson $\mathrm{V}_{8}$ which should dominate the colour formfactor of quarks $[60,65]$ (cf. Fig. 16). A phenomenological analysis of $w^{3}-\gamma-V$ mixing [66] yields for the isosinglet mass the lower bound

$\mathrm{M}_{\mathrm{V}}>250 \mathrm{GeV}$.

If one requires also $\mathrm{v}_{8}$-dominance of the quark colour form factor one obtains [65]

$\mathrm{MV}_{\mathrm{V}}>600 \mathrm{GeV}, \quad \mathrm{M}_{\mathrm{V}_{8}}>1 \mathrm{TeV}$.

There exists a vast literature on the phenomenology of "nearby compositeness", which has partly been reviewed in [67] and[68]. An interesting example are light pseudoscalar leptoquarks, which have been discussed by schrempp and Schrempp [69] as a possible explanation of three 


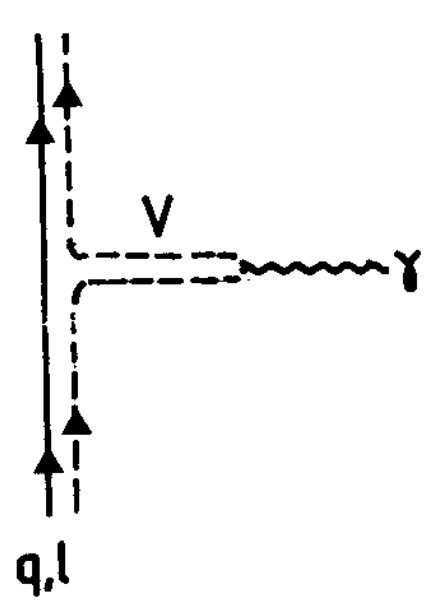

a)

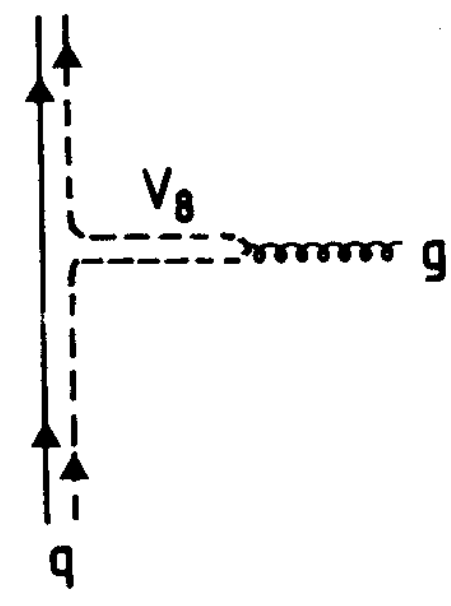

b)

Fig. 16.: V-dominance ot the isoscalar part of the electromagnetic form factor (a), and $\mathrm{V}_{8}$-dominance of the colour form factor (b).

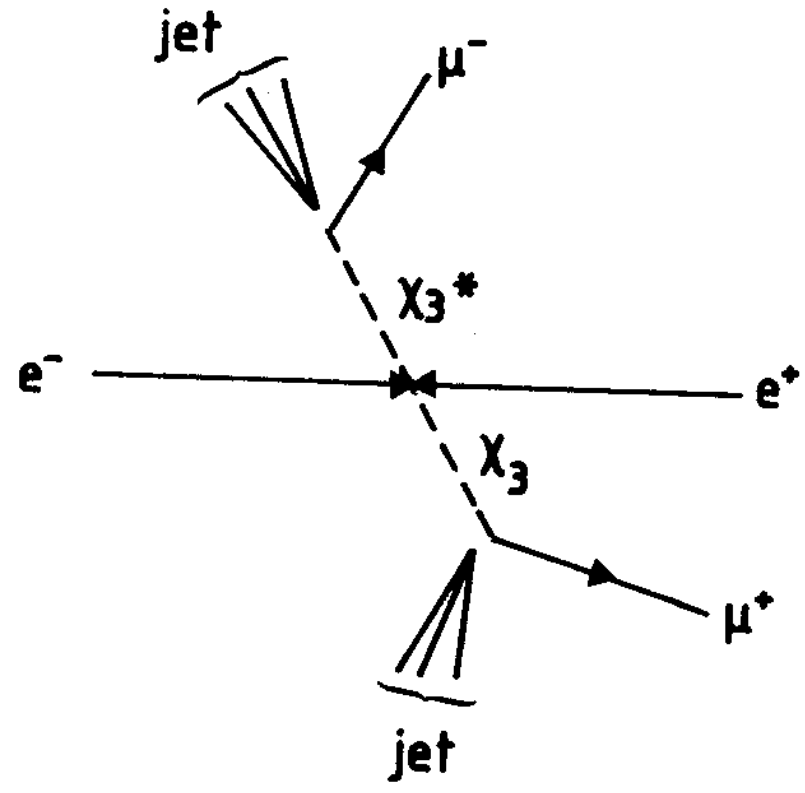

Fig. 17.: Leptoquark production and decay $1 n \in t$ 
striking events, containing two muons of opposite charge and two jets, which were reported by the CELLO collaboration [70] and the UA1 collaboration [71].

Light pseudoscalar leptoquarks (cf.(34))

$x_{3}=\left(h \ell^{*}\right) \sim \underline{3} \frac{2}{3}$

appear naturally in preon models of Pati-salam type as a consequence of the spontaneous symmetry breaking

$\mathrm{SU}(4)_{\mathrm{PS}} \rightarrow \mathrm{SU}(3)_{\mathrm{C}} \times \mathrm{U}(1)_{\mathrm{B}-\mathrm{L}} \cdot$

They are pseudo Goldstone bosons with a mass [69]

$m_{\chi_{3}}=O\left(\sqrt{\frac{\alpha}{\pi}} \Lambda\right)=O(40 \mathrm{GeV})$.

If there are separate leptoquarks for each generation, the leptoquark of the $\mu$-family will have the decay modes

$x_{3}+\mathrm{s \mu}^{+}$

$x_{3} \rightarrow \mathrm{Cv}_{\mu}$

The first decay mode could explain the CELLO event (cf.Fig.17) which would imply $\mathrm{m}_{\chi_{3}} \approx 20 \mathrm{GeV}$. As a consequence of this

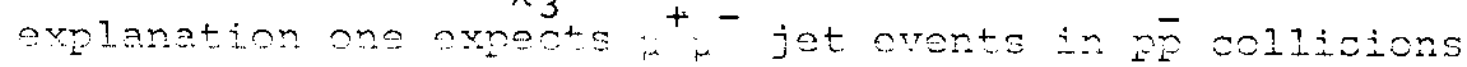
with an invariant mass of two $\mu$-jet combinations of $20 \mathrm{GeV}$ (cf. Fig.18). This signature as well as the expected production rates are indeed compatible [69] with two events reported by the UAI collaboration [71] (cf. Fig. 19). Obviously, no firm conclusions can be drawn from three events, but their qualitative features are very intriguing. 


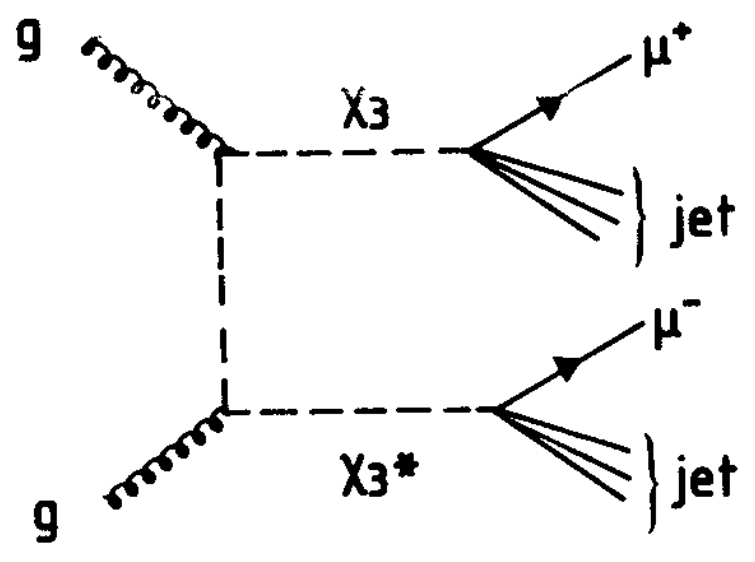

Fig. 18.: Leptoquark production and decay in p $\vec{p}$ collisions.

5. MASSLESS COMPOSITE SCALARS, FERMIONS AND VECTOR BOSONS

The main problem of quark-lepton substructure is the dynamics of bound states whose size is very small compared to their compton wave length, i.e.,

$\frac{1}{m_{f}} \frac{1}{\left\langle r_{f}\right\rangle}>>1$

Bound states familiar from atomic and nuclear physics, such as the deuteron or positronium, are of opposite type. They are non relativistic, and from the uncertainty relation $\frac{1}{\langle\mathrm{r}\rangle} \sim\langle\mathrm{p}\rangle=\mathrm{m}\left\langle\frac{\mathrm{V}}{\mathrm{c}}\right\rangle$ 


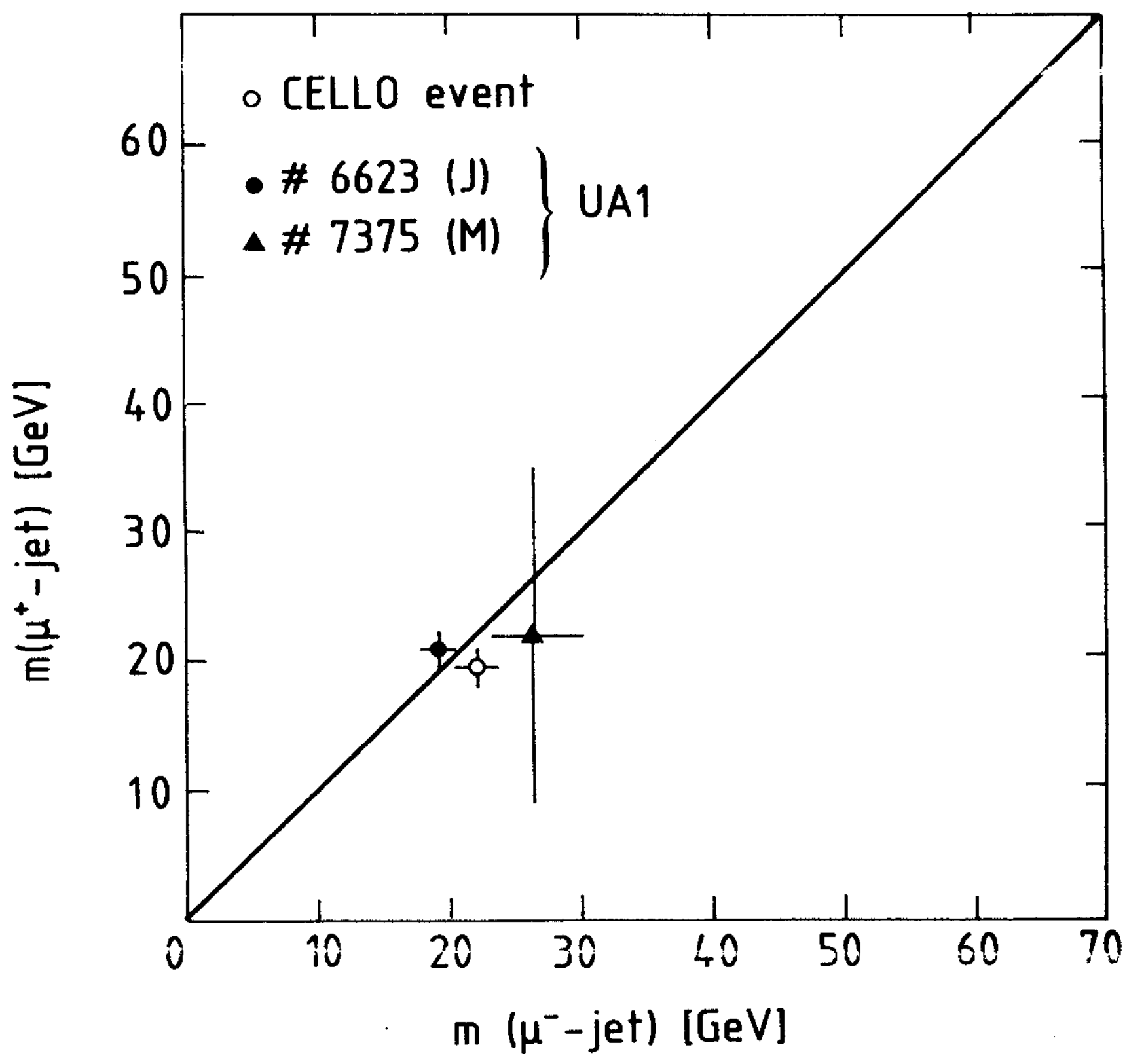

Fig. 19.: $m\left(\overline{\mu^{+}-j e t}\right)$ versus $m\left(\mu^{--j e t}\right)$ for the CELLO [70] and UA1[71] events (from ref.69). 
one obtains

$\frac{1}{\mathrm{~m}} \frac{1}{\langle\mathrm{r}\rangle}=\left\langle\frac{\mathrm{v}}{\mathrm{C}}\right\rangle\langle 1$.

Even the relativistic QCD bound states satisfy (78) (cf. table III), except for the pion which, as a pseudoGoldstone boson, plays a special role. It is clear from table III that, if quarks and leptons are composite, their structure is likely to be very different from the bound states we know.

Table III:Masses and sizes of some "elementary" particles (cf. [50], [72])

\begin{tabular}{lccc}
\hline particle & $\begin{array}{c}\text { mass } \\
\mathrm{m}[\mathrm{MeV}]\end{array}$ & $\begin{array}{c}\text { size } \\
\langle\mathrm{r}>\mathrm{fm}]\end{array}$ & $\frac{1}{\mathrm{~m}} \frac{1}{<\mathrm{r}\rangle}$ \\
\hline proton & 938 & 0.86 & 0.25 \\
pion & 135 & 0.75 & 2.0 \\
electron & 0.5 & $<4 \times 10^{-4}$ & $>10^{6}$ \\
neutrino & 0 & $>0$ & $\infty$ \\
\hline
\end{tabular}

A crucial ingredient in the description of (almost) massless fermions is chiral symmetry [73]. Let us consider the Lagrangian of a set of Dirac fermions $\psi^{i}=\left(\psi^{\alpha i}\right)$, $i=1 \ldots \mathrm{N}$, which interact with massless nonabelian vector bosons $\mathrm{V}_{\mu}^{\mathrm{A}}$ : 


$$
\begin{aligned}
L= & i \bar{\psi}_{i} \not \psi^{i}-m_{i} \bar{\psi}^{i}, \\
& \not{ }_{\beta}^{\alpha}=\gamma^{\mu}\left(\delta_{\beta}^{\alpha} \partial_{\mu}+i g \frac{1}{2}\left(\lambda^{A}\right){ }_{\beta}^{\alpha} v_{\mu}^{A}\right) .
\end{aligned}
$$

In the case of the gauge group su(3) the Lagrangian (79) corresponds to $Q C D$ with $N$ flavours. In terms of the chiral projections

$\psi_{\mathrm{R}}=\frac{1}{2}\left(1 \mp \gamma_{5}\right) \psi, \quad \gamma_{5}{\underset{\mathrm{L}}{\mathrm{R}}}=\mp_{\mathrm{R}}$,

the Lagrangian (79) reads

$L=i \bar{\psi}_{L i} \not \not \psi_{L}^{i}+i \bar{\psi}_{R i} \not \not \psi \psi_{R}^{i}-m\left(\bar{\psi}_{L i} \psi_{R}^{i}+\bar{\psi}_{R i} \psi_{L}^{i}\right)$

In the chiral limit $m \rightarrow 0$ the classical theory has the conserved currents

$J_{L(R) \mu}^{A}=\bar{\psi}_{L(R) i_{\mu}^{\gamma}}\left(T^{A}\right){ }_{j}^{i}{ }_{L(R)}^{j}, \quad \partial^{\mu} J_{L(R) \mu}^{A}=0$,

where $\mathrm{T}^{\mathrm{A}}$ are the generators of $\mathrm{U}(\mathrm{N})$; the corresponding charges

$Q_{L(R)}^{A}=\int a^{3} x J_{L(R) \circ}^{A}(x)$

generate the chiral symmetry $U(N){ }_{L} x U(N){ }_{R}$. In the quantum theory only the currents of the $S U(N){ }_{L} \times{ }^{S U(N)}{ }_{R} \times U(1)_{L+R}$ subgroup are conserved, whereas the $U(1)_{L-R}$ symmetry is broken due to instanton effects.

Massless composite particles are very special objects and, as we will discuss in the following, some of their 
properties can be determined from general theoretical. principles without solving the complete bound state problem.

\section{(i) Mass lesss_compositte_scalalar}

The only known composite particles, whose size is small compared to their compton wave length, are the pions. They are very light because they are pseudo-Goldstone bosons associated with the spontaneous breaking of chiral symmetry in QCD. It is an important consequence of their Goldstone nature that their self interactions at distances

$r>\frac{1}{\mathrm{f}_{\pi}} \sim \frac{1}{\Lambda_{Q C D}}$

are entirely determined by the symmetry breaking pattern. The QCD Lagrangian with two flavours $u_{L, R}$ and $d_{L, R}$ has the global symmetry

$\mathrm{G}=\mathrm{SU}(2)_{\mathrm{L}} \times \mathrm{SU}(2)_{\mathrm{R}} \times \mathrm{U}(1)_{\mathrm{V}}$,

which is generated by the charges $Q_{L, R}^{A}(A=1, \ldots, 3)$ and $Q_{V}$. One easily verifies that the vacuum expectation values

$\langle O|\bar{u} u| O\rangle=\langle O|\bar{d} d| O\rangle \sim \Lambda_{Q C D}^{3}$

break $G$ to the subgroup

$\mathrm{H}=\mathrm{SU}(2)_{\mathrm{V}} \times \mathrm{U}(1)_{\mathrm{V}}$

generated by the charges $T^{A}=Q_{L}^{A}+Q_{R}^{A}$ and $Q_{V}$. The vacuum is no longer invariant under the axial SU(2) ${ }_{\mathrm{A}}$ transformations generated by $x^{A}=Q_{R}^{A}-Q_{L}^{A}$. The corresponding Goldstone bosons are the $\mathrm{sU}(2)_{\mathrm{V}}$ triplet of pions which are created 
from the vacuum by the axial current:

$<0\left|\bar{q}(0) \gamma_{\mu} \gamma_{5} \frac{1}{2} \tau^{A} q(0)\right| \pi^{B}(p)>=i p_{\mu} \delta^{A B} f_{\pi}$

An effective Lagrangian for the pion self interactions at momentum transfers smaller than $f_{\pi}$ can be obtained by constructing first a nonlinear realization of the full chiral group $G$ on the fields $\pi^{A}(x)$ which is linear for the subgroup $\mathrm{H}$ [74]

$$
\begin{aligned}
& \frac{1}{i}\left[T^{A}, \pi^{B}\right]=\varepsilon_{A B C} \pi^{C}, \\
& \frac{1}{i}\left[X^{A}, \pi^{B}\right]=-f_{\pi}\left[\frac{1}{2}\left(1-\frac{1}{f_{\pi}^{2}} \vec{\pi}^{2}\right) \delta^{A B}+\frac{1}{f_{\pi}^{2}} \pi^{A} \pi^{B}\right] .
\end{aligned}
$$

The requirement of invariance under (88) leads to a nonpolynomial Lagrangian which is unique up to redefinition of the pion field :

$$
L_{\text {eff }}=\frac{1}{2\left(1+\frac{1}{f_{\pi}^{2}}{t^{2}}^{2}\right.} \partial^{\mu} A_{\partial_{\mu}} \pi^{A} .
$$

An expansion in powers of $1 / f_{\pi}^{2}$ yields interaction terms, where always two pions couple with derivatives, which is a consequence of the low-energy theorems for Goldstone particles.

\section{(ii) Massless composite fermions}

Spontaneously broken chiral symmetry leads to massless composite scalar particles as Goldstone bosons. Unbroken chiral symmetry requires massless composite fermions whose spectrum is strongly restricted through 't Hooft's consistency conditions $[6]$. 
Let us consider the vector and axial vector currents for a gauge theory of the type defined in (79),

$$
\begin{aligned}
& J_{\mu}^{A}(x)=\bar{\psi}_{i}(x) \gamma_{\mu}\left(T^{A}\right){ }_{j}^{i} j^{j}(x), \\
& J_{5 \mu}^{A}(x)=\bar{\psi}_{i}(x) \gamma_{\mu} \gamma_{5}\left(T^{A}\right) j_{j}^{j} j(x),
\end{aligned}
$$

and compute the 3-point functions of one axial and two vector currents [75]

$$
\begin{aligned}
\Gamma_{\mu \nu \lambda}^{A B C}\left(k_{1}, k_{2}\right) & =\int a^{4} x_{1} d^{4} x_{2} e^{i\left(k_{1} x_{1}+k_{2} x_{2}\right)}<O\left|T\left(J_{5 \mu}^{A}(O) J_{\nu}^{B}\left(x_{1}\right) J_{\lambda}^{C}\left(x_{2}\right)\right)\right| O> \\
& =a^{A B C}(R) \Gamma_{\mu \nu \lambda}\left(k_{1}, k_{2}\right),
\end{aligned}
$$$$
d^{A B C}(R)=\operatorname{tr}\left[T^{A}\left\{T^{B}, T^{C}\right\}\right]
$$

The tensor $d^{A B C}(R)$ depends on the representation $R$ of the fermions $\psi^{i}$ and is related to the corresponding tensor of the fundamental representation $R_{0}$ through the anomaly coefficient:

$$
d^{A B C}(R)=A(R) d^{A B C}\left(R_{0}\right)
$$

Note that the tensor $\mathrm{d}^{A B C}$ changes its sign, if the representation $\mathrm{R}$ is changed to its complex conjugate $\mathrm{R}^{*}$ :

$$
\begin{aligned}
\mathrm{d}^{\mathrm{n} 3 \mathrm{C}}\left(\mathrm{R}^{*}\right) & =(-)^{2} \operatorname{tr}\left[\mathrm{T}^{\mathrm{N}}\left\{\mathrm{T}^{\mathrm{B}}, \mathrm{T}^{\mathrm{C}^{*}}\right\}\right] \\
& =-\operatorname{tr}\left[\mathrm{T}^{\mathrm{AT}}\left\{\mathrm{T}^{\mathrm{BT}}, \mathrm{T}^{\mathrm{CT}}\right\}\right] \\
& =-\operatorname{tr}\left[\left\{\mathrm{T}^{\mathrm{C}}, \mathrm{T}^{\mathrm{B}}\right\} \mathrm{T}^{\mathrm{A}}\right]=-\mathrm{d}^{\mathrm{ABC}}(\mathrm{R}) .
\end{aligned}
$$

The 3-point function $\Gamma_{\mu \nu \lambda}^{A B C}$ can be computed perturbatively by evaluating the graphs shown in Fig. 20 . 

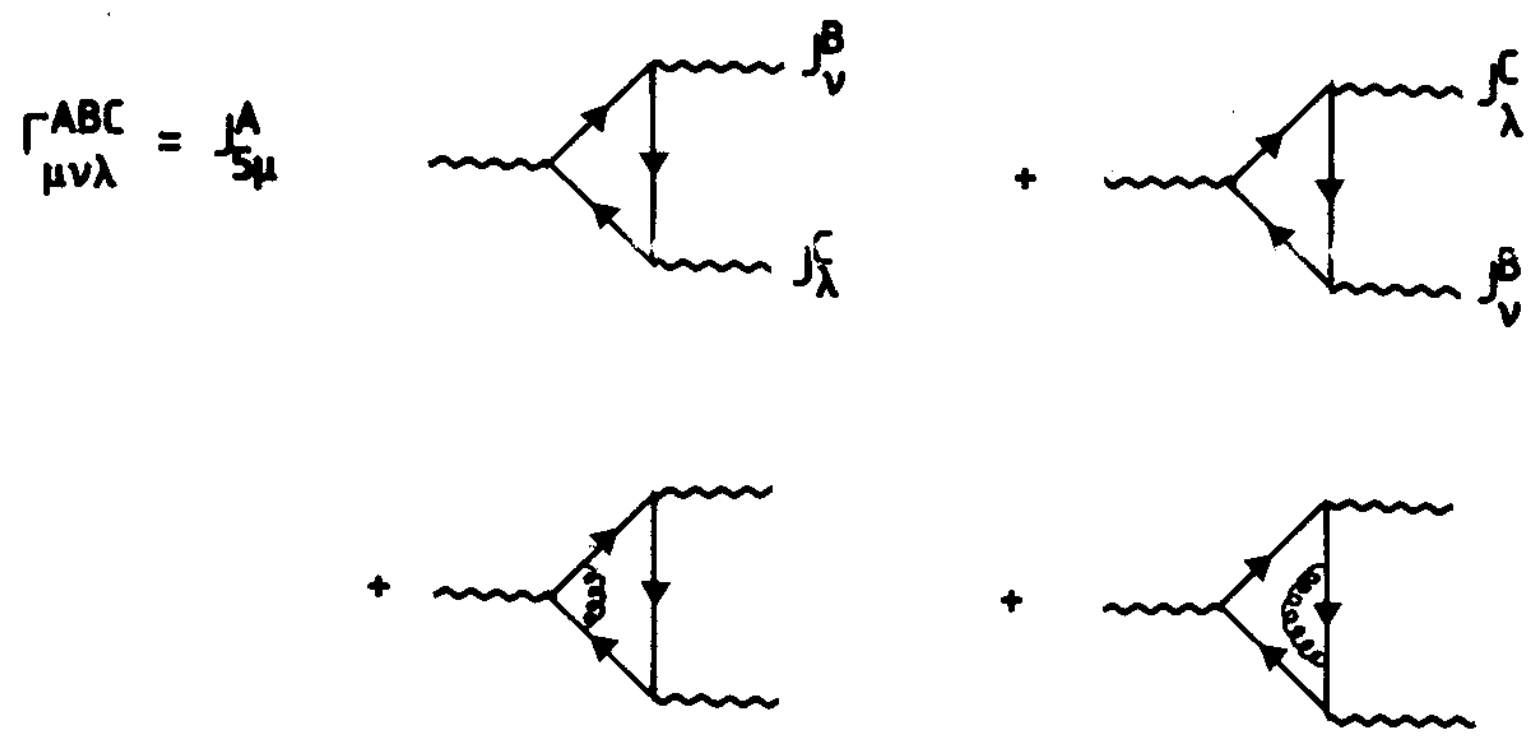

Fig. 20.: Contributions to the three-point function of one axial and two vector currents in perturbation theory (cf. (91)).

Classically the vector and axial vector currents (90) are conserved in the chiral limit,

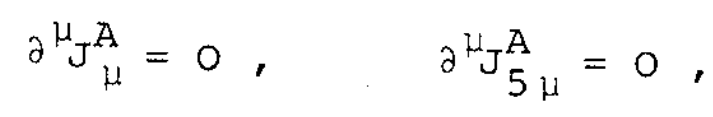

and thus one expects naively $\left(q=k_{1}+k_{2}\right)$ :

$k_{1}^{\nu} \underset{\mu \nu \lambda}{\Gamma_{\mu B C}}=k_{2}^{\lambda} \underset{\mu \nu \lambda}{\mathrm{ABC}}=0$

$q^{\mu} \Gamma_{\mu \nu \lambda}^{\mathrm{ABC}}=0$

It turns out, however, that there exists no regularization scheme, which is needed to give a meaning to the divergent 
quantity $\Gamma_{\mu \nu \lambda}^{\mathrm{ABC}}$, which preserves (95a) and (95b). Choosing (95a) to be valid, i.e., the conservation of the vector current, the divergence of the axial current does not vanish but is given by the Adler-Bell-Jackiw (ABJ) anomaly:

$q^{\mu} \Gamma_{\mu \nu \lambda}^{A B C}\left(k_{1} k_{2}\right) \underset{m \rightarrow 0}{\rightarrow} \frac{1}{2 \pi^{2}} d^{A B C}(R) \varepsilon_{\nu \lambda \rho \sigma} k_{1} \rho_{k_{2}^{\sigma}}$.

This result, which is obtained from a 1-loop calculation, is not modified through higher orders in perturbation theory, as Adler and Bardeen have shown. It is therefore natural to assume that (96) is an exact property of the theory

Let us consider now a preon theory with some exact chiral flavour symmetry $G$ and let $A\left(R_{p}\right)$ be an anomaly coefficient calculated from the fermionic preons. The composite fermions fall into two representations of $G$,
a) $R_{h}$,
$\mathrm{R}_{\mathrm{h}}=\mathrm{R}_{\mathrm{h}}^{*}$
b) $R_{\ell}$,
$\mathrm{R}_{\ell} \neq \mathrm{R}_{\ell}^{*}$

Fermions in the real representation $\mathrm{R}_{\mathrm{h}}$ are expected to have masses of the order of the hypercolour scale $\Lambda$, for fermions in the chiral representation $\mathrm{R}$ mass terms are forbidden.

Tf the ABT anomoly is on ovact nroperty of the theory,

it must be possible to calculate the anomaly coefficient

in terms of the fundamental as well as the composite fields. If $G$ is spontaneously broken the preon anomaly can be reproduced at the bound state level through the contribution of Fig.21, which involves the direct coupling of the Goldstone bosons to the broken currents. If $G$ is unbroken, the preon anomaly must be reproduced by the composite fermions. 


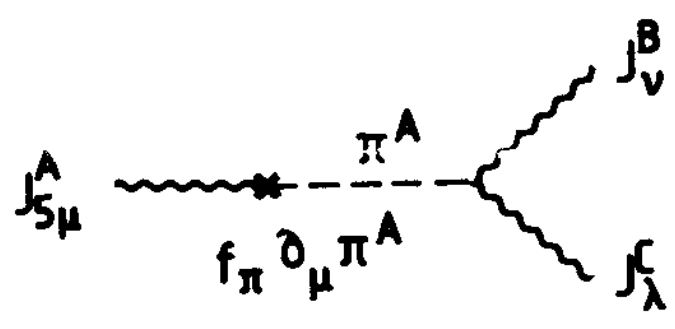

Fig. 21.: Direct coupling of Goldstone boson to current of spontaneously broken symmetry.

As the heavy fermions contained in $R_{h}$ do not contribute (cf. (92), (93)) we obtain 't Hooft's consistency condition [6]

$A\left(R_{p}\right)=A\left(R_{\ell}\right)$

which represents a very stringent constraint on preon theories.

It is very instructive to study the implications

of (98) for the preon model of Pati-Salam type described

in sec. 2. Associated with the fermionic preons $\psi^{\mathrm{p}}=$

$\psi_{\mathrm{L}}^{\mathrm{p}}+\psi_{\mathrm{R}}^{\mathrm{p}}(i=1,2)$ there are the following classically conserved currents $\left(\alpha=1, \ldots, \mathrm{n}\right.$ for $\left.\mathrm{SU}(\mathrm{n})_{\mathrm{HC}}\right)$ :

$\mathrm{J}_{\mu}^{(F)}(\mathrm{x})=\bar{\psi}_{\alpha}(\mathrm{x}) \gamma_{\mu} \psi^{\alpha}(\mathrm{x})$

$\mathrm{J}_{\mu}^{\mathrm{A}}(\mathrm{x})=\bar{\psi}_{\alpha}(\mathrm{x}) \gamma_{\mu} \frac{1}{2} \tau^{\mathrm{A}} \psi^{\alpha}(\mathrm{x})$

$\mathrm{J}_{5 \mu}^{\mathrm{A}}(\mathrm{x})=\bar{\psi}_{\alpha}(\mathrm{x}) \gamma_{\mu} \gamma_{5} \frac{1}{2} \tau^{\mathrm{A}} \psi^{\alpha}(\mathrm{x}) ;$

the charge $F=3 B+L$ corresponds to fermion number. In terms of composite quarks and leptons the currents (99) read 


$$
\begin{aligned}
& \left(i=1, \ldots, n_{F}\right): \\
& J_{\mu}^{(F)}(x)=\bar{q}_{i}(x) \gamma_{\mu} q^{i}(x)+\bar{\ell}_{i}(x) \gamma_{\mu} \ell^{i}(x), \\
& J_{\mu}^{A}(x)=\bar{q}_{i}(x) \gamma_{\mu} \frac{1}{2} \tau^{A} q^{i}(x)+\bar{\ell}_{i}(x) \gamma_{\mu} \frac{1}{2} \tau^{A} \ell^{i}(x), \\
& J_{5 \mu}^{A}(x)=\bar{q}_{i}(x) \gamma_{\mu} \gamma_{5} \frac{1}{2} \tau^{A} q^{i}(x)+\bar{l}_{i}(x) \gamma_{\mu} \gamma_{5} \frac{1}{2} \tau^{A} \ell^{i}(x)
\end{aligned}
$$

with

$$
\begin{array}{ll}
q^{i}=q_{L}^{i}+q_{R}^{i}, & \ell^{i}=\ell_{L}^{i}+\ell_{R}^{i}, \\
q_{R}^{i}=\left(\begin{array}{c}
u_{R}^{i} \\
d_{R}^{i}
\end{array}\right), & \ell_{R}^{i}=\left(\begin{array}{c}
v_{R}^{i} \\
e_{R}^{i}
\end{array}\right) .
\end{array}
$$

The anomaly coefficient for the 3-point function of $\mathrm{J}_{\mu}^{(\mathrm{F})}$, $J_{\nu}^{A}$ and $J_{5 \lambda}^{A}$ is easily obtained. As the preons $\psi^{p}$ are in the fundamental representation of $S U(2)$ with $F$-charge 1 , $A\left(R_{p}\right)$ is simply the multiplicity of $S U(2)$ doublets, i.e.,

$A\left(R_{p}\right)=n$

for the hypercolour group $\mathrm{SU}(\mathrm{n}) \mathrm{HC}$. In the same way one obtains for the composite fermions

$$
A\left(R_{\hat{i}}\right)=4 n_{E}
$$

because each generation contains $4 \mathrm{SU}(2)$ doublets. From 't Hooft's consisting condition (98) we thus obtain

$$
\mathrm{n}=4 \mathrm{n}_{\mathrm{f}}
$$


i.e., the matching of anomalies requires the hypercolour group SU(12) $\mathrm{HC}[27]$, if the bound state spectrum shall contain three generations of (almost) massless quarks and leptons! This result is not particularly encouraging. It means that the number of preons in the model of sec. 2 has to be larger than the number of quarks and leptons whose spectrum we want to explain. As a consequence of 't Hooft's consistency conditions it appears indeed unavoidable that a theory, whose bound-state spectrum contains massless fermions, is much more complicated than this sector of massless bound states. Thus we cannot hope for a simple preon theory. This, however, may not be too surprising since the theory for pions at distances larger than $1 / f_{\pi}$ is also much simpler than $\mathrm{QCD}$.

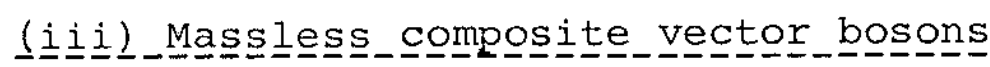

One could expect that a preon theory with massless bound states of spin o or $\frac{1}{2}$ may also lead to massless composite vector bosons. It has been shown, however, by Case and Gasiorowicz [76], and Weinberg and Witten [77] that massless vector bosons with global quantum numbers cannot exist. The theorem [77] reads as follows: "A theory that allows the construction of a Lorentz-covariant conserved four-vector current $J_{\mu}$ cannot contain massless particles of spin $>\frac{1}{2}$ with nonvanishing values of the conserved charge $\int \mathrm{J}_{0} \mathrm{~d}^{3} \mathrm{x} . "$

The proof is based on the comparison of two matrix elements of the current $J_{\mu}$, which correspond to "forward" and "backward scattering", in the limit of zero momentum. Due to Lorentz invariance one has

$$
\left\langle\mathrm{p}^{\prime}\left|J_{\mu}\right| \mathrm{p}\right\rangle \underset{\mathrm{p}^{\prime} \rightarrow \mathrm{p}}{\rightarrow} \mathrm{g} \frac{\mathrm{p}_{\mu}}{|\overrightarrow{\mathrm{p}}|}
$$


with $g \neq 0$ because the charge of the massless particle is assumed to be nonvanishing. On the other hand, helicity conservation implies

$\left\langle\mathrm{p}^{\prime}, \lambda\left|\mathrm{J}_{\mu}\right| \mathrm{p}, \lambda\right\rangle=0$

for $p=(|\vec{p}|, \vec{p}), p^{\prime}=(|\vec{p}|,-\vec{p})$ and $2 \lambda>1$. Considering the limit $|\vec{p}| \rightarrow 0$ leads to a contradiction between (104) and (105) for $\lambda>\frac{1}{2}$ which proves the proposition.

Similarly one can prove that particles with spin $j>1$ cannot exist in theories with a Lorentz-covariant energy-momentum tensor [77]. These theorems do not exclude Yang-Mills gauge theories or supergravity theories because these theories do not have Lorentz-covariant conserved currents or energy-momentum tensors, respectively.

We conclude this section by emphasizing again that some of the properties of composite massless particles can be determined from general theoretical principles without solving the difficult problem of relativistic bound states. Massless paxticles with nonvanishing quantum numbers with respect to global symmetries have to have spin o or $\frac{1}{2}$. Massless composite scalars can be obtained as Goldstone bosons; their self interactions at distances larger than the inverse symmetry breaking scale are completely determined by the pattern of symmetry breaking. Massless composite fermions have to satisfy 't Hooft's consisting conditions, which appear to lead unavoidably to rather complicated preon theories. 


\section{QUASI-GOLDSTONE FERMIONS}

Interesting candidates for a theory of quark-lepton substructure are supersymmetric (SUSY) confining gauge theories. Supersymmetric preon models [78-82] have been suggested in order to avoid the "naturalness problem" [4-6] for scalar preons whose presence appears to be necessary in phenomenologically acceptable models. Furthermore supersymmetric theories provide new mechanisms to generate massless composite fermions: supersymmetry may protect chiral symmetry from being dynamically broken [83-85], a spontaneous breakdown of extended supersymmetry can lead to Goldstone fermions [86], or quasi-Goldstone fermions [87] can appear as SUSY partners of Goldstone bosons due to the spontaneous breaking of global symmetries in supersymmetric theories.

Let us consider, for example, a SUSY confining gauge theory, such as SQCD, with some global chiral symmetry G. The bound state spectrum of such a theory will unavoidably contain massless composite fermions because either

(i) G is unbroken, which implies massless "baryons" (cf. $\sec 5(i i))$, or

(ii) G is spontaneously broken to $\mathrm{H}$ with unbroken SUSY, which yields quasi-Goldstone fermions as SUSY partners of "pions", or

(iii) SUSY is spontaneously broken with $G$ broken or unbroken, which leads at least to one massless fermion, the Goldstino.

The building blocks of supersymmetric gauge theories are chiral superfields $x_{a}=\left(\tilde{\chi}_{a}, \eta_{L a}\right)$, which contain complex scalars and weyl fermions as physical degrees of freedom, and vector superfields $v^{A}=\left(\lambda_{L}^{A}, v_{\mu}^{A}\right)$ containing Weyl fermions and massless vector fields [88]. In strongly interacting confining theories it is likely that a global 
symmetry $G$ of the Lagrangian is spontaneously broken to a subgroup $H$ through vacuum expectation values of the lowest components of composite chiral and vector superfields,

$$
\begin{aligned}
& <O\left|x_{a} x_{b}\right| O>\sim \Lambda_{H C}^{2}, \\
& <O\left|\bar{x}_{a} e^{2 g V} x_{b}\right| O>\approx \Lambda_{H C}^{2},
\end{aligned}
$$

where $\Lambda_{\mathrm{HC}}$ is the hypercolourscale. Due to supersymmetry the massless composite Goldstone bosons will be part of chiral superfields $\phi_{i}=\left(\tilde{\phi}_{i}, \psi_{\mathrm{L} i}\right)$.

A natural question to ask is whether the Goldstone mechanism in a supersymmetric theory could be the dynamical origin of composite quarks and leptons which are (almost) massless with respect to their substructure scale $\Lambda$. In such a picture masses of scalar quarks and leptons much smaller than $\Lambda$ could arise from the breaking of supersymmetry and some explicit breaking of the original global symmetry G, for instance through gauge interactions, which would turn the massless Goldstone bosons into massive pseudo-Goldstone bosons (cf. Fig.22). The masslessness of the quasi-Goldstone fermions could be guaranteed by some chiral symmetry contained in the unbroken subgroup $\mathrm{H} C \mathrm{G}$ [89]. In order to realize this idea, one has to solve a group theoretical problem: the unbroken subgroup $\mathrm{H}$ has to be identified with a physical group which contains at laset the group of unhroken aruae interactions.

$\mathrm{H}>\mathrm{SU}(3)_{\mathrm{C}} \times \mathrm{U(1)}{ }_{\mathrm{EM}} \times \ldots$;

furthermore, $\mathrm{H}$ must be embedded into a larger group $\mathrm{G}$ such that the broken generators in $G / H$ have the quantum numbers of quarks and leptons with respect to $\mathrm{H}$. 


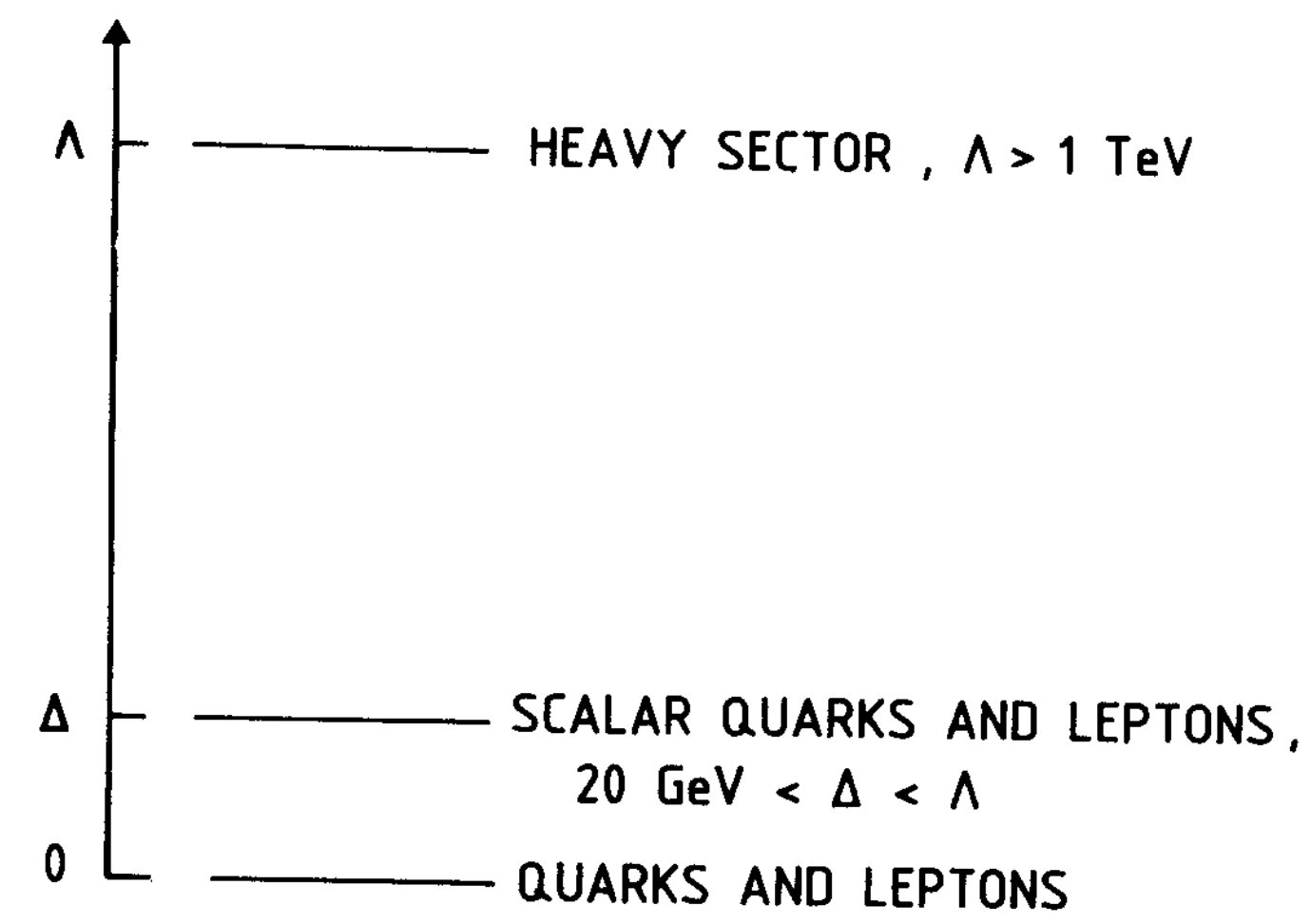

Fig. 22.: Qualitative picture of mass spectrum in supersymmetric preon models.

A simple example is the coset space $U(6) / S U(2) \times U(4)$ $[82,89-91]$, where the $S U(2)$ and $S U(4)$ subgroups are identified as weak isospin and Pati-salam symmetry (cf. sec.2). From the adjoint representation of $U(6)$,

$\mathrm{U}(6)=\quad\left(\begin{array}{lrc}\mathrm{U}(4) & 1 & \left(4,2^{*}\right) \\ \hdashline\left(4^{*}, 2\right) & 1 & \mathrm{U}(2)\end{array}\right)$,

one reads off that the Goldstone bosons (GB's) corresponding to $\mathrm{U}(6) / \mathrm{U}(2) \mathrm{xU}(4)$ transform as

$\mathrm{GB}^{\prime} \mathrm{s} \sim\left(4,2^{*}\right)+\left(4^{*}, 2\right)$,

i.e., they form a real representation of $U(2) \times U(4)$. Their 
SUSY partners, however, the quasi-Goldstone fermions (QGF's), may be chosen to transform as a chiral representation of $\mathrm{U}(2) \times \mathrm{U}(4)$,

$\mathrm{QGF}^{\prime} \mathrm{s} \sim\left(4,2^{*}\right)$.

The Goldstone bosons and the quasi-Goldstone fermions form together a chiral multiplet $\phi_{p}^{a} \sim\left(4,2^{*}\right)(a=1, \ldots, 4$; $\mathrm{p}=1,2)$ which corresponds to the left-handed sector of one supersymmetric quark-lepton family. The possibility to obtain chiral fermions $[89,92]$ as SUSY partners of real Goldstone bosons is of crucial importance in model building [93-96]. The coset space $U(6) / U(2) \times U(4)$ can also be associated with the real representation $\left(4,2^{*}\right)+\left(4^{*}, 2\right)$ of $\mathrm{QGF}^{\prime} \mathrm{s}[90]$. It is a dynamical question which case is realized.

The coset space necessary to accommodate one quartet of left-handed quarks and leptons is $U(6) / U(2) \times U(4)$. The study of explicit models as well as general arguments [97-99] show, however, that the unbroken subgroup of $U(6)$ is smaller than $U(2) \times U(4)$. In particular the breaking of $\mathrm{U}(2)$ to $\mathrm{SU}(2)$ gives rise to a Goldstone chiral multiplet, the "novino" [93], which contains only one Goldstone boson and one additional scalar (quasi-Goldstone boson). The presence of their SU(2) $x$ SU(4) neutral chiral multiplet $\phi$ has important consequences for the self-interactions of all Goldstone multiplets $\phi_{p}^{a}$ and $\phi[59,93,100]$.

The interactions of Goldstone superfields at distances $r<\frac{1}{f}$, where $f$ is the mass scale of symmetry breaking, are described by supersymmetric o-models [102]. They have the special property that the complex scalar fields can be interpreted as coordinates of a Kähler manifold. This means that the metric, which determines the $\sigma$-model Lagrangian, can locally be expressed as the second derivative of a potential: 


$$
\begin{gathered}
L_{s}=\partial^{\mu \tau_{i}^{*} g_{i j}}\left(\tilde{\phi}^{*}, \tilde{\phi}\right) \partial_{\mu} \tilde{\phi}_{j}, \\
g_{i j}\left(\tilde{\phi}^{*}, \tilde{\phi}\right)=\frac{\partial 2}{\partial \tilde{\phi}_{i}^{*} \partial \tilde{\phi}_{j}} K\left(\tilde{\phi}^{*}, \tilde{\phi}\right) .
\end{gathered}
$$

The supersymmetric extension of the Lagrangian for the scalar fields takes a simple form in terms of the Kähler potential $\mathrm{K}[103]$ :

$$
\begin{aligned}
L & =\int d^{4} \theta k(\bar{\phi}, \phi) \\
& =L_{k i n}-\frac{1}{f^{2}} \tilde{\phi}_{i}^{* \sim} \phi_{i} \partial^{\mu \psi_{j}^{*}{ }_{j} \tilde{\phi}_{j}}-\frac{1}{2 f^{2}} \bar{\psi}_{L i} \gamma^{\mu} \psi_{L i} \bar{\psi}_{L j} \gamma_{\mu} \psi_{L j}+\ldots
\end{aligned}
$$

The residual interactions of the quasi-Goldstone fermions are of current-current type which is of interest in models with composite $W$-bosons $[90,91]$. This is in contrast to Goldstone fermions where the residual interactions involve derivatives [86]:

$$
L=L_{k i n}-\frac{1}{2 f} 4 \bar{\psi}_{i} \gamma^{\mu} \partial \nu_{j} \bar{\psi}_{i} \gamma_{\nu} \partial_{\mu} \psi_{j}+\ldots .
$$

The Kähler potentials for the coset spaces based on $U(6)$, which we discussed above, are exactly known. For $U(6) / U(2) \times U(4)$ one has [103]

$K=f^{2} \ln \left(\operatorname{aet}\left(f_{y}^{p}+\frac{1}{f^{2}}-p_{a}^{p} a\right)\right)$

In the case of $U(6) / S U(2) \times U(4)$ the Kähler potential is given by $[100,101]$

$$
K=F\left[e^{i / f_{1}(\phi-\bar{\phi})} \operatorname{det}\left(\delta_{q}^{p}+\frac{1}{f_{2}^{2}} \bar{\phi}_{a}^{p_{q}}{ }_{q}^{a}\right)\right]
$$


where $\mathrm{F}$ is an arbitrary function up to the constraints

$$
\frac{\partial^{2} F}{\partial \bar{\phi} \partial \phi}=1, \frac{\partial^{2} F}{\partial \bar{\phi}{ }_{a}^{p} \partial \phi} \frac{b}{q}=\delta_{b}^{a} \delta_{p}^{q} .
$$

The arbitrariness of the Kähler potential (115) compared to (114) is due to the presence of one quasi-Goldstone boson in the case of $\mathrm{U}(6) / \mathrm{SU}(2) \times \mathrm{U}(4)$ which implies that the metric of the Kähler manifold is not determined entirely by the $U(6)$ symmetry.

The coset space $U(6) / S U(2) \times U(4)$, which accommodates one quartet of left-handed quarks and leptons, is the main building block of more realistic models which are based on $U(6) \times U(6) '$. In such models the $W$-bosons may be composite $[91,95]$ or fundamental $[89,95,96]$. In the latter case the local $\mathrm{SU}(2)_{\mathrm{W}} \times \mathrm{U}(1)_{\mathrm{Y}}$ symmetry is broken through the technicolour type breaking [104] of $\mathrm{SU}(2) \times \mathrm{SU}(2)$ ' to the diagonal SU(2) subgroup. The class of $U(6) \times U(6)$ ' models has interesting theoretical and phenomenological features, but so far no completely satisfactory preon model has been found. With respect to the question of family replication it is an interesting possibility [91] that the number of quark-lepton generations is a consequence of the dimension of the hypercolourgroup and 't Hooft's anomaly matching conditions.

\section{COMPOSITE HIGGS FIELDS AND EXCEPTIONAL COSET SPACES}

As we saw in the previous section the effective Lagrangian for Goldstone superfields is a supersymmetric o-model, i.e., a pure D-term. The absence of a superpo- 
tential raises the question how fermion masses can be generated in such theories. In this section we will discuss a nonperturbative mechanism for fermion mass generation [105], which is a consequence of supersymmetry breaking. The questions of supersymmetry breaking and its implications have been discussed in a similar context by Masiero and Veneziano [106] and Achiman, Aoyama and van Holten [107].

Let us consider a simple SUSY $\sigma$-model for "leptons" based on the coset space $U(3) \times U(2) / U(2) x U(1)^{2}$. The unbroken subgroup $U(2) \times U(1)^{2}$ contains the electroweak gauge group $S U(2)_{W} \times U(1)_{Y}$ and the global $U(1)_{\ell}$ of lepton number.

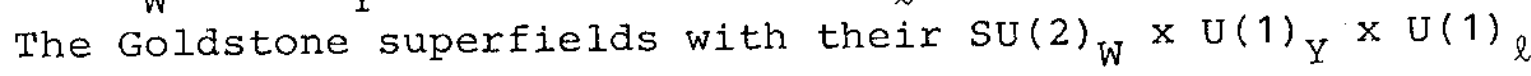
quantum numbers are [105]

$L^{i} \sim\left(2,-\frac{1}{2}, 1\right), E_{C} \sim(1,1,-1), S \sim(1,0,0) ;$

they correspond to a left-handed doublet of leptons, a left-handed positron and an additional singlet containing one quasi-Goldstone boson.

An exact Kähler potential for the coset space $U(3) \times U(2) /$ $/ \mathrm{U}(2) \mathrm{x} \mathrm{U}(1)^{2}$, which can be obtained by standard methods, reads

$$
\begin{aligned}
K_{O} & =f^{2}+f(S+\bar{S})+\bar{L}_{i} L^{i}+\bar{E}_{C} E_{C}+\bar{S} S \\
& +\frac{1}{f^{2}} \bar{L} L^{i} \bar{E}_{E} E_{C}\left[\left(1+\frac{1}{f} S\right)\left(1+\frac{1}{f} \bar{S}\right)\right]-1 .
\end{aligned}
$$

Adding a scalar mass $\Delta<<$ for the scalars $\tilde{\mathrm{L}}^{i}$ and $\tilde{\mathrm{E}}_{c}$, and gauging $\mathrm{SU}(2)_{\mathrm{W}} \times \mathrm{U}(1)_{\mathrm{Y}}[104,108]$ one obtains (the anomalies of the gauged currents are irrelevant in the following discussion and will be absent in a realistic theory including quarks): 


$$
\begin{aligned}
L_{\sigma} & =\int d^{4} \theta\left[\bar{L}_{i}\left(e^{2 g W-g^{\prime} B}\right) i_{j}^{L^{j}+\bar{E}_{C}} 2 g^{\prime} B_{E_{C}}\right](1-\delta)+\vec{S} S \\
& +\frac{1}{f^{2}} \bar{L}_{i} \bar{E}_{C}\left(e^{2 g W+g^{\prime} B}\right) j_{j}{ }^{j} E_{C}\left[\left(1+\frac{1}{f} S\right)\left(1+\frac{1}{f} \bar{S}\right]^{-1},\right. \\
\delta & =\Delta^{2} \theta \theta \bar{\theta} \bar{\theta}, \quad \Delta<f .
\end{aligned}
$$

The Lagrangian (119) is similar to the supersymetric Nambu-Jona Lasinio (SNJL) model [84]. Therefore one may expect that the soft supersymmetry breaking in (119) induces a further symmetry breaking through vacuum expectation values of composite fields describing bound states of $I^{i}$ and $E_{C}[109]$. In analogy to the SNJL model such composite fields can be found by rewriting (119) with the aid of auxiliary superfields $\mathrm{H}_{1}$ and $\mathrm{H}_{2}$ :

$$
\begin{aligned}
& L_{t}=\int d^{4} \theta\left\{\left[\overline { L } _ { i } \left(e^{2 g W-g^{\lambda} B_{j}}{ }_{j}^{i} L^{j}+\bar{E}_{C} e^{\left.2 g^{\prime} B_{E_{C}}\right](1-\delta)+\bar{s} s}\right.\right.\right. \\
& \left.+\overrightarrow{\mathrm{H}}_{1 i}\left(e^{2 g W+g^{\prime} B}\right){ }_{j}^{i_{H}}{ }_{i}^{j}\right\} \\
& +\int \mathrm{d}^{2} \theta\left[\mathrm{fH} \mathrm{i}_{1}^{\mathrm{H}_{2 i}}+\mathrm{H}_{1}^{\dot{i}_{\mathrm{H}}} \mathrm{H} \mathrm{S}+\mathrm{L}^{\dot{i}} \mathrm{H}_{2 i} \mathrm{E}_{\mathrm{C}}\right]+\mathrm{c} \cdot \mathrm{c} .
\end{aligned}
$$

In (120) $\mathrm{H}_{2}$ appears without kinetic term, i.e., it acts as a Lagrange multiplier field. Inserting the corresponding constraint for $\mathrm{H}_{1}$ in (120) yields the old Lagrangian (119). Therefore, although $\mathrm{H}_{1}$ does appear with kinetic term in

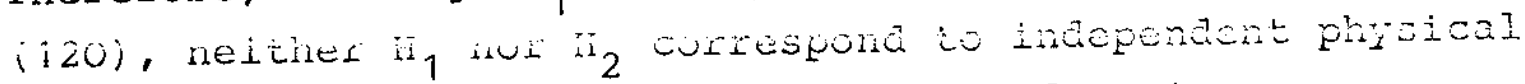
degrees of freedom. From the equations of motion one obtains:

$$
\begin{aligned}
& \mathrm{H}_{1}^{i}=-\frac{1}{\mathrm{f}}\left(1+\frac{1}{\mathrm{f}} \mathrm{S}\right)^{-1} \mathrm{~L}_{\mathrm{E}_{C}}, \\
& \mathrm{H}_{2 \mathrm{i}}=\frac{1}{\mathrm{f}^{2}}\left(1+\frac{1}{\mathrm{f}} \mathrm{S}\right)^{-1}\left(-\frac{1}{4}\right) \bar{D}^{2}\left[( 1 + \frac { 1 } { \mathrm { f } } \overline { \mathrm { S } } ) ^ { - 1 } \overline { \mathrm { L } } _ { j } \overline { \mathrm { E } } _ { \mathrm { C } } \left(\mathrm{e}^{\left.\left.2 \mathrm{gW}+\mathrm{g}^{\prime} \mathrm{B}\right)_{i}^{j}\right]} .\right.\right.
\end{aligned}
$$


It is remarkable that $\mathrm{H}_{1}$ and $\mathrm{H}_{2}$ have the quantum numbers of the Higgs fields in the supersymmetric standard model [110], and that they interact with the other chiral superfields through a superpotential. It is indeed a non-trivial consequence of the kähler potential (118) that the auxiliary fields are chiral and not vector superfields!

As in the SNJL model one finds that one-loop radiative corrections generate a kinetic term for $\mathrm{H}_{2}$ (cf. Fig.23):
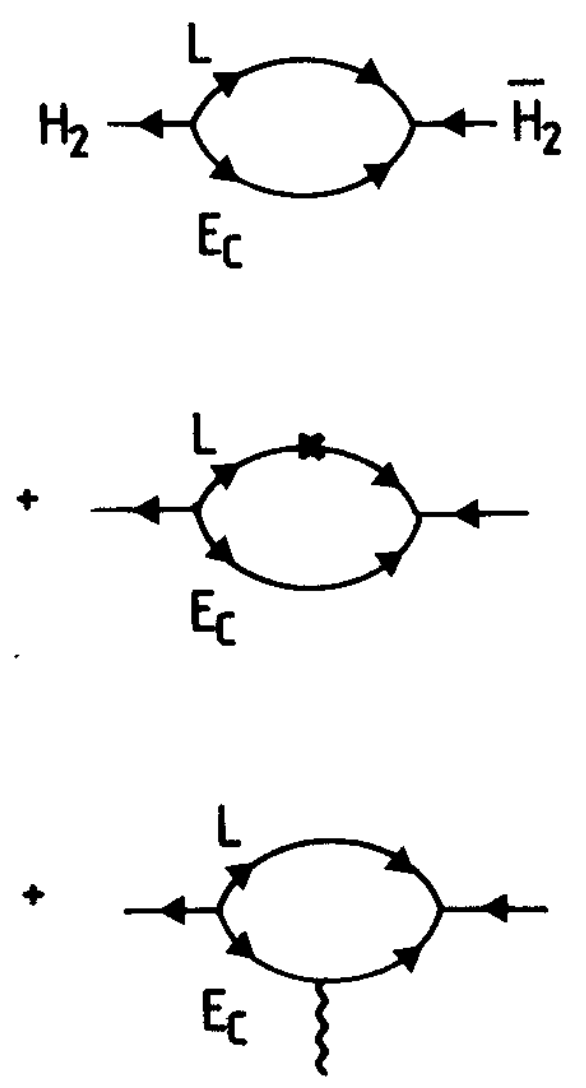

B
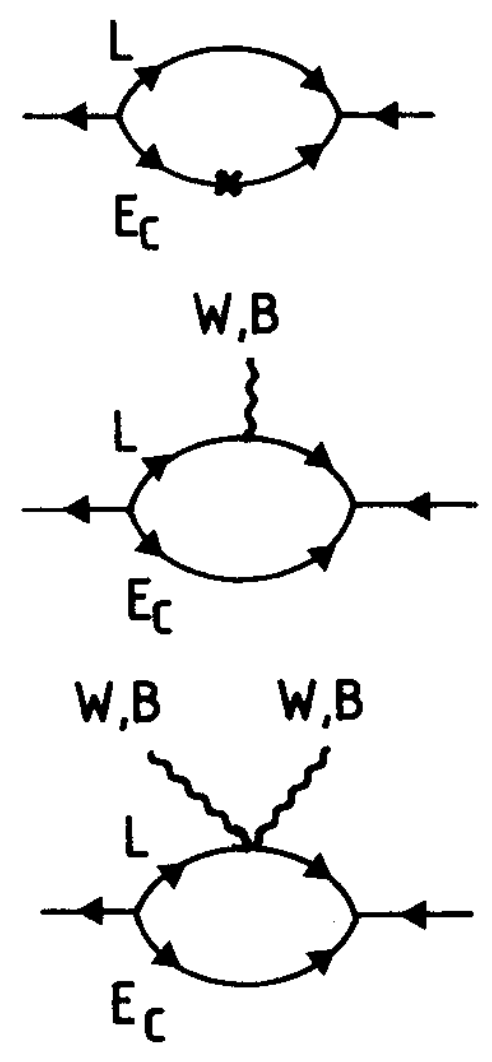

Fig. 23.: Supergraphs contributing to the kinetic term of $\mathrm{H}_{2} \cdot$ 
$L_{\ell}=z \int d^{4} \theta \overline{\mathrm{H}}_{2}^{i}\left(e^{-2 g W-g^{\prime} B}\right){ }_{j}^{i_{H} j}(1+2 \delta)+o\left(\frac{1}{\ln \frac{f^{2}}{\Delta}}\right)$,

with $z=\frac{1}{16 \pi^{2}}\left(\ln \frac{f^{2}}{\Delta^{2}}+o(1)\right)$.

Therefore in the one-loop approximation, which may be justifiable by means of a $\frac{1}{N}$ expansion in an extended version of the model, we obtain the Higgs fields $\mathrm{H}_{1}$ and $\mathrm{H}_{2}$ as bound states of the fields $L^{i}, E_{C}$ and $S$. The supersymmetry breaking term in (122) corresponds to a negative mass squared for $\breve{H}_{2}$. This leads to a vacuum expectation value of $\mathrm{H}_{2}$ and thereby via the superpotential in (120) to fermion masses whose existence has already been anticipated as an infrared cutoff in some of the one-loop integrals of Fig.23. Given the composite Higgs fields, the described mechanism for $S U(2)_{W} \times U(1)_{Y}$ breaking is similar to the radiative symmetry breaking proposed by Ibánez and Ross $[111]$.

From the Lagrangian (120), (122) the entire mass spectrum of the model can be calculated in the standard manner [110] as function of the o-model scale $f$ and the SUSY breaking scale $\Delta$. Minimization of the scalar potential yields the vacuum expectation values

$\left\langle\tilde{\mathrm{H}}_{1}\right\rangle=\left(\begin{array}{l}0 \\ v \\ v_{1}\end{array}\right), \quad \sqrt{2}<\tilde{\mathrm{H}}_{?}>=\left(\begin{array}{c}0 \\ v_{2}\end{array}\right)$,

with

$v_{1}^{2}=\frac{2\left(\frac{1}{4}\left(g^{2}+g^{\prime 2}\right) z-1\right) z \Delta^{2}}{\frac{1}{2}\left(g^{2}+g^{\prime 2}\right) z-1}$ 
$\mathrm{V}_{2}^{2}=\frac{\left(g^{2}+g^{\prime 2}\right) z^{2} \Delta^{2}}{2\left(\frac{1}{2}\left(g^{2}+g^{\prime 2}\right) z-1\right)}$

The physical states are the vector bosons $W^{ \pm}, z$ and $\gamma$, the neutrino $\nu$ and the Dirac fermions $e, h^{+}, h^{-}, h$ and $h^{\prime}$ (which are combinations of Weyl fermions from $\mathrm{H}_{1}, \mathrm{H}_{2}{ }^{\prime} \mathrm{L}, \mathrm{E}_{\mathrm{C}}$ '

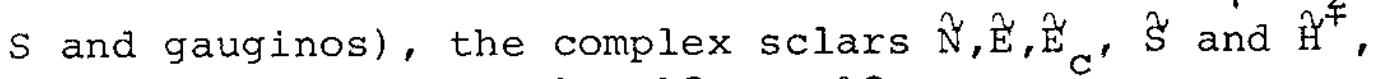
and the real scalars $\tilde{H}_{0}, \tilde{H}_{1}^{O}$ and $\mathrm{H}_{2}^{\circ}$. The corresponding masses are given by

$\left(\cos \theta_{W}=\frac{g}{\sqrt{g^{2}+g{ }^{2}}}, \cos \phi=\frac{v_{2}}{\sqrt{v_{1}^{2}+v_{2}^{2}}}\right):$

$M_{W}^{2}=\frac{1}{2} g^{2}\left(v_{1}^{2}+v_{2}^{2}\right), \quad M_{Z}^{2}=\frac{1}{2}\left(g^{2}+g^{\prime 2}\right)\left(v_{1}^{2}+v_{2}^{2}\right), \quad M_{\gamma}^{2}=0$,

$\mathrm{m}_{\mathrm{h}^{+}}=\sqrt{2} \sin \phi \mathrm{M}_{\mathrm{W}^{\prime}}, \quad \mathrm{m}_{\mathrm{h}^{-}}=\sqrt{2} \cos \phi \mathrm{M}_{\mathrm{W}}$,

$\mathrm{m}_{\mathrm{h}}=\sqrt{2} \Delta, \quad \mathrm{m}_{\mathrm{h}^{\prime}}=\mathrm{M}_{\mathrm{Z}}$,

$m_{e}=\sqrt{2} \Delta \cos \phi, \quad m_{v}=0$,

$M^{2} \tilde{N}=\Delta^{2}\left(1+2 \cos ^{2} \phi\right), \quad M_{\tilde{E}}^{2}=\Delta^{2}\left(1+4 \cos ^{2} \phi \sin ^{2} \theta\right)$,

$\mathrm{M}_{\text {है }}^{2}=\Delta^{2}\left(1+2 \cos ^{2} \phi\left(1-2 \sin ^{2} \theta_{W}\right)\right), \quad M_{S}^{2}=2 \Delta^{2} \cos ^{2} \phi$,

$\mathrm{M}_{\mathrm{H}}^{2}+=\mathrm{M}_{\mathrm{W}}^{2}-2 \Delta^{2}, \quad \mathrm{M}_{\mathrm{H}_{\mathrm{O}}}^{2}=0$,

$\mathrm{M}_{\mathrm{H}}^{2} \mathrm{O}_{1,2}^{\mathrm{O}}=\Delta^{2}\left[\mathrm{x} \pm\left(\frac{\mathrm{x}\left(\mathrm{x}^{2}-5 \mathrm{x}+8\right)}{\mathrm{x}-1}\right)^{1 / 2}\right], \quad \mathrm{x}=\frac{1}{2}\left(\mathrm{~g}^{2}+\mathrm{g}^{2}\right) \mathrm{Z}=\frac{\mathrm{M}_{\mathrm{Z}}^{2}}{2 \Delta^{2}} \geq 2$. 
So far we have only discussed a model for "leptons". A more realistic theory including quarks and more than one generation will have a much more complicated set of composite Higgs fields. In such a theory the one-loop graphs of the type shown in Fig. 23 will be dominated by the t-quark, i.e., the multiplet which couples most strongly to the composite Higgs fields. Identifying $m_{e}$ with the $t$-quark mass yields indeed a phenomenologicaliy consistent mass spectrum. Choosing for instance as input $\mathrm{M}_{\mathrm{W}}=81 \mathrm{GeV}, \mathrm{M}_{\mathrm{Z}}=92 \mathrm{GeV}$ and $\mathrm{m}_{\mathrm{e}}=38 \mathrm{GeV}$, in order to $\mathrm{fix}$ the parameters $\Delta, f$ and $\sin ^{2} \theta_{W^{\prime}}$ one obtains for the remaining masses (in $\mathrm{GeV}$ ) from equs. (124):

$$
\begin{aligned}
& \mathrm{m}_{\mathrm{h}}+=66, \quad \mathrm{~m}_{\mathrm{h}}-=94, \quad \mathrm{~m}_{\mathrm{h}}=46 \text {, } \\
& m_{h^{\prime}}=92, \quad m_{\nu}=0, \quad M_{N}=50 \text {, } \\
& \mathrm{M}_{\mathrm{E}}^{\sim}=41, \quad \mathrm{M}_{\mathrm{C}}^{\widetilde{E_{C}}}=43, \quad \mathrm{M}_{\mathrm{S}}=38 \text {, } \\
& \mathrm{M}_{\mathrm{H}^{+}}=67, \quad \mathrm{M}_{\mathrm{H}^{\circ}}^{\sim 0}=0, \quad \mathrm{M}_{\mathrm{H}_{1}}=82 \text {, } \\
& \mathrm{M}_{\mathrm{H}_{2}^{\circ}}^{\circ}=42 \cdot
\end{aligned}
$$

The $\sigma$-model scale $f$ can be expressed in terms of the Fermi constant $G_{F}$ and the supersymmetry breaking scale $\Delta$ (cf. (122), (123)):

$\ln \frac{\Sigma^{2}}{\Delta^{2}}=8 \pi=\frac{v_{i}^{2}+v_{2}^{2}}{s^{2}}=\frac{2 \sqrt{2} 2}{\Delta^{2} G_{F}}$.

From (126) it is clear that $\Delta$ has to be of the order of the Fermi scale $G_{F}^{-1 / 2}$, and that $f$ is naturally much larger than $\mathrm{G}_{F}^{-1 / 2}$, i.e., the substructure scale has to be identified with the unification mass $M_{G U T}$ or the Planck mass $M_{P L}$ rather 
than the Fermi scale (in fact for the mass spectrum (125) one obtains $f>M_{P L}$, but equ. (126) is likely to be modified in a more realistic theory).

The possibility that Higgs fields may be described as bound states of quark and leptons has been considered before by Harari and Seiberg [112]. More recently Napoly has studied dynamical symmetry breaking through fermion condensates in a grand-unified so(10) model [113]. If quarks and leptons are themselves composite, vacuum expectation values of composite Higgs fields correspond to higher dimensional preon condensates which are currently also being studied by Mizrachi and Peccei in connection with fermion mass generation [114]. Of course, Higgs fields should be considered as bound states of quarks and leptons only in an effective sense, i.e., in the same way as nucleons [115] and $p$-mesons [116] can be obtained from a $\sigma$-model of pions.

What is the correct $\sigma$-model for (at least) three generations of quarks and leptons? The most interesting coset spaces are clearly those involving exceptional groups $[87,90,92,117-121]$, and $\sigma$-models based on $\mathrm{E}_{7}[118]$, $E_{6}[119]$ and $E_{8}[121,122]$ have already been constructed. From the SU(5) decomposition of the adjoint representations [ 123 ] it is abvious that a spontaneous symmetry breaking of $E_{6}, E_{7}$ or $E_{8}$ will yield Goldstone multiplets with the quantum numbers of quarks and leptons:

$$
\begin{aligned}
& E_{6}: \underline{78}=\underline{24}+\underline{5}+\underline{5}+2 \times(\underline{10}+\underline{10})+4 \times \underline{1}, \\
& E_{7}: \underline{133}=\underline{78}+3 \times(\underline{5}+\underline{5})+\underline{10}+\underline{10}+5 \times \underline{1}, \\
& E_{8}: \underline{248}=\underline{133}+6 \times(\underline{5}+\underline{5})+2 \times(\underline{10}+\underline{10})+15 \times \underline{1},
\end{aligned}
$$


where $24, \underline{78}, \underline{133}$ and $\underline{248}$ denote the adjoint representations of $\mathrm{SU}(5), \mathrm{E}_{6}, \mathrm{E}_{7}$ and $\mathrm{E}_{8}$ respectively.

In order to accomodate three quark-lepton families in a coset-space $\mathrm{G} / \mathrm{H}$ the dimension of $\mathrm{G}$ has to be sufficientIy large. As each generation contains 15 Weyl fermions one has

$n_{G} \geq n_{H}+2 \times 15 \times 3=n_{H}+90$.

Thus for $\mathrm{H} \supset \mathrm{SU}(5) \mathrm{E}_{7}$ and $\mathrm{E}_{8}$ are possible choices for $\mathrm{G}$ whereas for $\mathrm{H}>\mathrm{SO}(10)$ or $\mathrm{E}_{6}$ only $\mathrm{E}_{8}$ is large enough. Appropriate Kählex manifolds can be found by means of a recipe recently given by Bordemann, Forger and Römer [124]. It states that $\mathrm{G} / \mathrm{H}$ is a Kähler manifold, if $\mathrm{H}$ takes the form $\mathrm{H}=\mathrm{H}^{\prime} \mathrm{x} U(1)^{\mathrm{P}}$ where $\mathrm{H}^{\prime}$ is a semi-simple subgroup of $G$ which one obtains from the Dynkin diagram of $G$ by crossing out $\mathrm{p}$ roots; $\mathrm{U}(1)^{\mathrm{P}}\left(\mathrm{p}=\operatorname{rank}(\mathrm{G})-r a n k\left(\mathrm{H}^{\prime}\right)\right.$ ) are the $U(1)$ factors of $G$ which are not contained in $H^{\prime}$. Some examples for $G=E_{6}, E_{7}, E_{8}$ are shown in Fig. 24 .

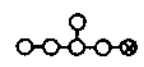

a)

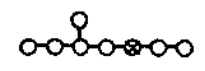

c)

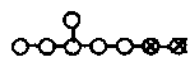

e)

\section{$0000-000$}

b)

$0-0$ ㅇ-o-0

d)

Fig. 24.: Dynkin diagrams for the Kähler manifolds $E_{6} /$ $/ \mathrm{SO}(10) \times U(1)(a), E_{7} / \mathrm{SU}(5) \times \mathrm{SU}(3) \times \mathrm{U}(1)$ (b) $, E_{8} / S O(10) \times S U(3) \times U(1)(c), E_{8} / E_{6} \times S U(2) \times$ $x U(1)$ (d), $E_{8} / E_{6} \times U(1)^{2}$ (e). 
Given a Kähler manifold $G / H$ one has to find the Ginvariant complex structures $\Omega$ which determine the H-quantum numbers of the chiral superfields of the supersymmetric $\sigma$-models. The complex structures can be easily found by decomposing the adjoint representation of $\mathrm{G}$ with respect to $\mathrm{H}$ and selecting those roots of $\mathrm{G} / \mathrm{H}$ whose charges with respect to a $U(1)$ factor contained in $U(1)^{p}$ have the same sign.

For the five cases of Fig. 24 one finds

a) $E_{6} / \mathrm{SO}(10) \times U(1)$ [119]:

$$
\Omega=\underline{16}^{1} .
$$

b) $\mathrm{E}_{7} / \mathrm{SU}(5) \times \mathrm{SU}(3) \times \mathrm{U}(1) \quad[118]$ :

$$
\Omega=(5,1)^{6}+(\overline{5}, 3)^{4}+(10,3)^{2} .
$$

c) $\mathrm{E}_{8} / \mathrm{SO}(10) \times \mathrm{SU}(3) \times \mathrm{U}(1)[121,122]$ :

$$
\Omega=(16,3)^{1}+(\overline{16}, 1)^{3}+(1,3)^{4}+(10, \overline{3})^{2} .
$$

d) $\mathrm{E}_{8} / \mathrm{E}_{6} \times \mathrm{SU}(2) \times \mathrm{U}(1) \quad[124]$ :

$$
\Omega=(27,2)^{1}+(\overline{27}, 1)^{2}+(1,2)^{3} .
$$

e) $E_{8} / E_{6} \times U(1)^{2}[124]:$

$$
\Omega=\underline{27}^{i, i}+27^{i,-i}+\underline{27}^{2,0}+\underline{1}^{2, i}+\underline{1}^{2, \cdots+}+1^{0,2} .
$$

In the last case there also exists a unique complex structure, although $\mathrm{H}$ contains two $\mathrm{U}(1)$ factors.

$$
\text { Only the coset spaces b) and c) contain three families. }
$$
As in case b) $\mathrm{SU}(5)$ cannot be gauged because of anomalies, the 
coset space c) appears to be the most promising candidate for a description of quarks and leptons. Its characteristic feature is the prediction of a fourth mirror family.

It is conceivable that the investigation of supersymmetric $\sigma$-models for quarks and leptons will lead to an understanding of their quantum numbers (and the prediction of some specific new particles), and that the nonrenormalizable interactions of the Goldstone multiplets, together with gauge interactions and supersymmetry breaking, can eventualiy explain the quark-lepton mass spectrum. It is certainly a very remarkable phenomenon, that the coset spaces of successive exceptional groups (cf. [126]), which include the interesting gauge groups, contain precisely the quantum numbers of quarks and leptons. We conclude this section by listing this "exceptional sequence":

$$
\begin{aligned}
& E_{3}=S U(3) \times S U(2) \quad 0-0^{O} \\
& \mathrm{E}_{4}=\mathrm{SU}(5) \\
& \text { O-O } \stackrel{0}{\circ} \\
& E_{n+1} / E_{n} \times \cup(1): \\
& E_{5}=S O(10) \\
& 0-0-0-0 \\
& (3,2)+\text { c.c. } \\
& 10+\text { c.c. } \\
& 16(=\overline{5}+10+1)+\text { c.c. } \\
& \mathrm{E}_{6} \\
& 0-0-0-0-0 \\
& E_{7} \\
& 0-0-\dot{O}-0-0-0 \\
& 27(=16+10+1)+\text { c.c. } \\
& 56(=27+\overline{27}+1+1)+1+\text { c.c. }
\end{aligned}
$$




\section{CONCLUSIONS}

In these lectures we have discussed various aspects of quark-lepton substructure. Starting from the standard gauge theory of strong and electroweak interactions we have argued that the replication of quark-lepton generations and the hierachical pattern of their masses suggest a further substructure of matter. We have studied a representative preon model of Pati-Salam type and examined experimental bounds on the compositeness scale $\Lambda$. It is phenomenologically possible, although unlikely, that $\Lambda$ is as small as the Fermi scale $\mathrm{G}_{\mathrm{F}}{ }^{-1 / 2} \sim 300 \mathrm{GeV}$. In this case the $W$-vector bosons of the weak interactions would be composite and their effective Lagrangian would be a massive Yang-Mills theory.

A central question of quark-lepton substructure is the dynamics of massless bound states. The only known composite particles, whose mass is small compared to their inverse size, are the pions which are the pseudo-Goldstone bosons of the spontaneously broken axial isospin symmetry of QCD. Massless composite fermions which originate from an asymptotically free preon theory have to satisfy 't Hooft's anomaly matching conditions. An apparently unavoidable consequence of these consistency conditions is that preon theories have to be very complicated and that the number of preons is generally larger than the number of massless composite fermions. A further important constraint is that massless particles with global quantum numbers have to have spin o or $\operatorname{spin} \frac{i}{2}$.

The most interesting candidates for a theory of quarklepton substructure are confining supersymmetric gauge theories which contain naturally massless composite fermions. They can appear because of unbroken chiral symmetries 
or as a consequence of symmetry breaking: Goldstone fermions arise from the spontaneous breaking of supersymmetry, and quasi-Goldstone fermions from the spontaneous breaking of global symmetries in the case of unbroken supersymmetry. The group structure of supersymmetric preon models is similar to non-supersymmetric models, the amount of complexity is even larger in the supersymmetric case.

The main ingredients of preon models have been borrowed from $Q C D$ : the concepts of a confining, asymptotically free hypercolour force and of chiral symmetry. It is disappointing that so far attempts based on these ideas have not led to a "standard composite model" and that in particular no convincing explanation has been found for the main puzzle of the standard model, the replication of quark-lepton families. Therefore we conclude that the QCD-motivated approach described in most of these lectures is presumably inappropriate and that novel ideas are needed.

In connection with supersymmetric preon models supersymmetric o-models play an important role. It is conceivable that these $\sigma$-models are more interesting than the renormalizable theories which notivate them. The existence of Kähler manifolds whose linear symmetries are the relevant gauge groups and whose coordinates carry quark-lepton quantum numbers is a remarkable phenomenon. It is an intriguing possibility that the role of supersymmetric o-models for the dynamics of quarks and leptons could be analogous to the role of ordinary o-models for the drnamics of hadrons before the discovery of QCD.

\section{ACKNOWLEDGEMENTS}

These lectures are an extended version of lectures given at he Herbstschule für Hochenergiephysik, Maria-Laach, September 1984. I thank Professors J.K. Bienlein, H. Mitter 
and W. Plessas for the invitations to Maria-Laach and Schladming. I am grateful to U. Ellwanger, O. Napoly, M.G. Schmidt, D. Wyler, T. Yanagida and, especially, R.D. Peccei for a stimulating collaboration on some aspects of quark-lepton substructure.

\section{REFERENCES}

1. S.L. Glashow, Nucl. Phys. 22 (1961) 579.

S. Weinberg, Phys. Rev. Lett. 19 (1967) 1264.

A. Salam, in Elementary Particle Theory, ed. N. Svartholm (Almqvist and Wiksell, Stockholm, 1968) 367.

2. J.C. Pati and A. Salam, Phys. Rev. D10 (1974) 275.

3. H. Georgi and S.L. Glashow, Phys. Rev. Lett. 32 (1974) 438.

4. K.G. Wilson, Phys. Rev. D3 (1971) 1818.

5. L. Susskind, Phys, Rev. D2O (1979) 2619.

6. G. 't Hooft, in Recent Developments in Gauge Theories, Cargèse 1979, eds. G. 't Hooft et al. (Plenum, New York and London).

7. For a review and an extensive list of references, see: L. Lyons, Progress in Particle and Nuclear Physics 10 (1983) 227; Oxford preprint 2/84 (1984).

8. For an introduction, see, for instance: G.G. Ross, Grand unified theories (The Benjamin/Cummings Publishing Company, Inc., 1985).

9. For a recent review, see:

L. wyler, Lectures glver at the siscuela inexicana de Particulas y Campos, Oaxtebec, Mexico, December 1984, ETH preprint (1985).

10. There exists a vast literature on this subject. For recent reviews, see:

G. Ecker, Acta Phys. Pol. B15 (1984) 179; B. Stech, in Proceedings of the Europhysics Topical Conference 
on Flavor Mixing in Weak Interactions, Erice, 1984, ed. by L.L. Chau (Plenum Press, New York, 1984).

11. L. Wolfenstein, Phys. Rev. Lett. 51 (1983) 1945.

12. P.H. Frampton and C. Jarlskog, Phys. Lett. 154B (1985) 421.

13. J. Gasserand H. Leutwylex, Phys. Rep. 87C (1982) 77.

14. J.C. Pati and A. Salam, ref.2, footnote 7 .

15. O.W. Greenberg and C.A. Nelson, Phys. Rev. D10 (1974) 2567.

16. K. Matumoto, Prog. Theor. Phys. 52 (1974) 1973.

17. J.C. Pati, A. Salam and J. Strathdee, Phys. Lett. 59B (1975) 265 .

18. M.E. Peskin, in Proc. of the tepton Photon Symposium, Bonn University, ed. W. Pfeil (1981), p.880.

19. R.D. Peccei, in Proc. of the Fourth Topical Workshop on Proton-Antiproton Collider Physics, Bern, eds. H. Hänni and J. Schacher (1984), p.483.

20. H. Terazawa, in Proc. of the XXII International Conference on High Energy Physics, Leipzig, eds. A. Meyer and

E. Wieczorek (1984), p.63.

21. H. Harari, Phys. Lett. 86B (1979) 83 .

22. M.A. Shupe, Phys. Lett. 86B (1979) 87.

23. H. Harari and N. Seiberg, Phys. Lett. 98B (1981) 269; Nucl. Phys. B204 (1982) 141.

24. I. Bars, Nuc1. Phys. B208 (1982) 77.

25. B. Schrempp and F. Schrempp, Nucl. Phys. B231 (1984) 109; ibid. B242 (1984) 203.

26. H. Georgi and D.B. Kaplan, Phys. Lett 145B (1984) 216; H. Georgi, Phys. Lett. 151B (1985) 57.

27. R. Barbieri, A. Masiero and R.N. Mohapatra, Phys. Lett. 105B (1981) 369 .

28. L. Abbott and E. Farhi, Phys. Lett. 101B (1981) 69.

29. O.W. Greenberg and J. Sucher, Phys. Lett. 99B (1981) 339 .

30. H. Fritzsch and G. Mandelbaum, Phys. Lett. 102B (1981) 369 . 
31. R. Casalbuoni and R. Gatto, Phys. Lett. 103B (1981) 113.

32. H. Terazawa and K. Akama, Phys. Lett. 96B (1980) 276.

33. H. Harari, Phys. Rep. 104 (1984) 159.

34. For a more complete discussion, see:

S. Yamada, in Proc. of the XXII International Conference on High Energy Physics, Leipzig, eds. A. Meyer and E. Wieczorek (1984) p.72.

35. E.J. Eichten, K.D. Lane and M.E. Peskin, Phys. Rev. Lett. 50 (1983) 811 .

36. M. Abolins et al., in Proc. of the 1982 summer study on Elementary Particles and Fields, Snowmass, eds. R. Donaldson et al. (Amer. Inst. Phys. N.Y., 1983) p.274.

37. R. Rückl, Phys. Lett. 129B (1983) 363.

38. R. Rückl, Nucl. Phys. B234 (1984) 91.

39. T. Maehara and T. Yanagida, Prog. Theor. Phys. 61 (1979) 1434 .

40. R.N. Cahn and H. Harari, Nucl. Phys. B176 (1980) 135.

41. G.L. Kane and R. Thun, Phys. Lett. 94B (1980) 513.

42. S. Dimopoulos and J. Ellis, Nucl. Phys. B182 (1981) 505.

43. I. Bars, in Proc. Moriond Workshop on Quarks, Leptons and Supersymmetry, ed. J. Tran Thanh Van (1982) p.541.

44. C.N. Leung, S.T. Love and S. Rao, preprint FermilabPub-84/74-T (1984).

45. Ch. Kopper, Phys. Lett. 155B (1985) 409.

46. W. Buchmuller and D. Wyler, in preparation.

47. R. Barbieri, L. Maiani and R. Petronzio, Phys. Lett. 96B (1980) 63 .

48. S. Brodsky and S.D. Drell, Phys. Rev. D22 (1980) 2236.

49. T. Kinoshita and W.B. Lindquist, Phys. Rev. Lett. $\underline{47}$ (1981) 1573; T. Kinoshita, B. Nižíc, and Y. Okamoto, Phys. Rev. Lett. 52 (1984) 717.

50. Review of Particle Properties, Rev. Mod. Phys. $\underline{56}$ (1984) No.2, Part II. 
51. O.W. Greenberg, R.N. Mohapatra and S. Nussinov, Phys. Lett. 148B (1984) 465 .

52. J.D. Bjorken, Phys. Rev. D19 (1979) 335 .

53. P.Q. Hung and J.J. Sakurai, Nucl. Phys. B143 (1978) 81.

54. R. Kögerler and D. Schildknecht, preprint CERN-TH 3231 (1982).

55. H. Fritzsch, R. Kögerler and D. Schildknecht, Phys. Lett. 114B (1982) 157.

56. H.G. Dosch, M. Kremer and M.G. Schmidt, Phys. Lett. 137B (1984) 88; Z. Phys. C26 (1985) 569.

57. V.A. Matveev, M.E. Shaposhnikov and A.N. Tavkhelidze, Moscow preprint P-0325 (1983).

58. S. Narison, Montpellier preprint PM 84/23 (1984).

59. W. Buchmiiller and M.G. Schmidt, Nucl. Phys.B285 (1985) 230 .

60. B. Schrempp and F. Schrempp, preprint DESY 84-055 (1984).

61. N.M. Kroll, T.D. Lee and B. Zumino, Phys. Rev. 157 (1967) 1376.

62. J.J. Sakurai, Acta Phys. Aust. Suppl. XXIV (1982) 275.

63. P. Chen and J.J. Sakurai, Phys. Lett. 110B (1982) 481.

64. H. Fritzsch and G. Mandelbaum, Phys. Lett. 109B (1982) 224 .

65. W. Buchmililer, Phys. Lett. 145B (1984) 151.

66. M. Kuroda, D. Schildknecht and K.-H. Schwarzer, Bielefeld preprint BI-TP 84/22 (1984).

67. R.D. Peccei, in Proc. of the ECFA-CERN Workshop, Lausanne, ed. M. Jacob, (1984) p.329.

68. F.M. Renard, Montpelliex preprint PM/84/4 (1984).

69. B. Schrempp and F.Schrempp, Phys. Lett. 153B (1985) 101.

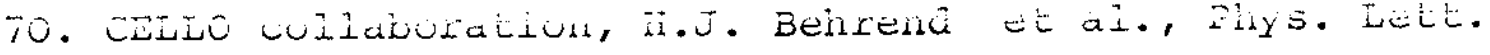
141B (1984) 145 .

71. UA1 collaboration, G. Arnison et al., Phys. Lett. 155B (1985) 442 .

72. O. Dumbrajs et al., Nuc1. Phys. B216 (1983) 304 .

73. For a thorough review of "chiral symmetry and chiral 
symmetry breaking", see: M.E. Peskin, Lectures given at the 1982 Los Houches summer School of Theoretical Physics, preprint SLAC-PUB-3021 (1982).

74. S. Weinberg, Phys. Rev. 166 (1968) 1568.

75. See, for instance, C. Itzykson and J.-B. Zuber, Quantum Field Theory (McGraw-Hill Inc., 1980).

76. K.M. Case and S. Gasiorowicz, Phys. Rev. 125 (1962) 1055. 77. S. Weinberg and E. Witten, Phys. Lett. 96B (1980) 59. 78. J.-M. Gérard, J. Govaerts, Y. Meurice and J. Weyers, Phys. Lett. 116B (1982) 29; Nucl. Phys. B234 (1984) 138.

79. R. Barbieri, Phys. Lett. 121B (1983) 43.

80. J.C. Pati and A. Salam, Nucl. Phys. B214 (1983) 109.

81. Y.J. Ng and B.A. Ovru, Phys. Lett. 125B (1983) 147; Phys. Rev. D29 (1984) 138.

82. F. Bordi, R. Casalbuoni, D. Dominici and R. Gatto, Phys. Lett. 127B (1983) 419 .

83. H.P. Nilles, Phys. Lett. 112B (1982) 455.

84. W. Buchmüller and S.T. Love, Nucl. Phys. B204 (1982) 213. 85. G. Veneziano, Phys. Lett. 124B (1983) 357.

86. W.A. Bardeen and V. Višnjić, Nucl. Phys. B194 (1982) 422.

87. W. Buchmüller, S.T. Love, R.D. Peccei and T. Yanagida, Phys. Lett. 115B (1982) 233; W. Buchmiuller, R.D. Peccei and T. Yanagida, Phys. Lett. 124B (1983) 67.

88. We use the conventions of J. Wess and J. Bagger, Supersymmetry and Supergravity (Princeton University Press, Princeton, 1983).

89. R. Barbieri, A. Masiero and G. Veneziano, Phys. Lett. $128 B$ (1983) 493 .

yo. W. Buchmuler, K.u. reccei ana i. raraglad, lvuci. triys. B227 (1983) 503 .

91. O.W. Greenberg, R.N. Mohapatra and M. Yasue, Phys. Lett. 127B (1983) 419; Phys. Rev. Lett. 51 (1983) 1737.

92. C.L. Ong, Phys. Rev. D27 (1983) 911.

93. W. Buchmüller, R.D. Peccei and T. Yanagida, Nucl. Phys. B231 (1984) 53 . 
94. W. Lerche and D. Lüst, Nucl. Phys. B244 (1984) 157.

95. W. Buchmïllex, R.D. Peccei and T. Yanagida, Nucl. Phys. B244 (1984) 186.

96. A. Masiero, R. Pettoxino, M. Roncadelli and G. Veneziano, preprint CERN-TH. $\underline{4166}$ (1985)

97. W. Lerche, Nucl. Phys. B238 (1984) 582.

98. G. Shore, Nucl. Phys. B248 (1984) 123.

99. W. Lerche, thesis, Munich 1985.

100. T. Kugo, S. Uehara and T. Yanagida, Phys. Lett. 147B (1984) 321.

101. M. Bando, T. Kuramoto, T. Maskawa and S. Uehara, Phys. Lett. 138B (1984) 94 .

102. For a review, see: J. Bagger, Lectures given at the NATO Advanced Study Institute on Supersymmetry, Bonn 1984, preprint SLAC-PUB-3461 (1984).

103. B. Zumino, Phys. Lett. 87B (1979) 203.

104. A. Buras and W. SXominski, Nucl. Phys. B223 (1983) 157.

105. W. Buchmüller and U. Ellwanger, preprint CERN-TH 4113 (1985).

106. A. Masiero and G. Veneziano, preprint CERN-TH 3950 (1984).

107. Y. Achiman, S. Aoyama and J.W. van Holten, Phys. Lett. 15OB (1985) 153 .

108. J. Bagger and E. Witten, Phys. Lett. 118B (1982) 103.

109. W. Buchmüller and U. Ellwanger, Nucl. Phys. B245 (1984) 237 .

110. For a review, see: H.P. Nilles, Phys. Rep. 110 (1984).

111. L.E. Ibáñez and G.G. Ross, Phys. Lett. 110B (1982) 215.

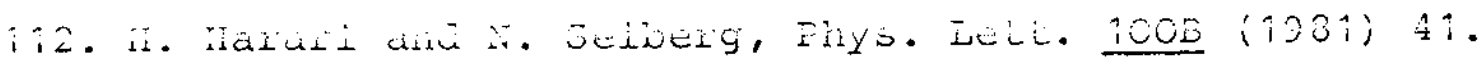

113. O. Napoly, Saclay preprint SPhT-84-51 (1984).

114. R.D. Peccei, Lectures given at CERN, February 1985.

115. T.H.R. Skyrme, Proc. Roy. Soc. A260 (1961) 127;

E. Witten, Nucl. Phys. B223 (1983) 433.

116. M. Bando, T. Kugo, S. Uehara, K. Yamawaki and T. Yanagida, Phys. Rev. Lett. $\underline{54}$ (1985) 1215 . 
117. C.L. Ong, Phys. Rev. D27 (1983) 3044 .

118. T. Kugo and T. Yanagida 134B (1984) 313.

119. Y. Achiman, S. Aoyama and J.W. van Holten, Phys. Lett. 141B (1984) 64; Wuppertal preprint WU B 85-2 (1985).

120. L.E. Ibáñez, Phys. Lett. 150B (1985) 127.

121. C.L. Ong, preprint SLAC-PUB-3459 (1984).

122. S. Irié and Y. Yasui, Tohoku preprint TU/85/278 (1985).

123. R. Slansky, Phys. Rep. 79 (1981) 1.

124. M. Bordemann, M. Forger and H. Römer, preprint CERN-TH $\underline{4111}$ (1985).

125. W. Buchmüller and O. Napoly, preprint CERN-TH 4197 (1985)

126. D.I. Olive, in Unification of the Fundamental Particle Interactions II, Erice 1981, edited by J. Ellis and S. Ferrara (Plenum Press, New York, 1983). 